\title{
Semi-implicit finite strain constitutive integration and mixed strain/stress control based on intermediate configurations
}

\author{
P. Areias ${ }^{\mathrm{a}, \mathrm{e}, *}$, T. Rabczuk ${ }^{\mathrm{b}}$, J. César de Sá ${ }^{\mathrm{c}}$, J.L. Alves ${ }^{\mathrm{d}}$ \\ ${ }^{a}$ Department of Physics, University of Évora, Colégio Luís António Verney, Rua Romão Ramalho, 59, 7002-554 Évora, Portugal \\ ${ }^{\mathrm{b}}$ Institute of Structural Mechanics, Bauhaus-University Weimar, Marienstraße 15, 99423 Weimar, Germany \\ ${ }^{\mathrm{c}}$ Mechanical Engineering Department, Faculty of Engineering, University of Porto, Rua Dr. Roberto Frias, s/n, 4200-465 Porto, Portugal \\ ${ }^{\mathrm{d}}$ Mechanical Engineering Department, School of Engineering, University of Minho, Campus de Azurém, 4804-533 Guimarães Portugal \\ e ICIST/CERIS, Instituto Superior Técnico, Lisboa, Portugal
}

\section{A R T I C L E I N F O}

\section{Article history:}

Received 22 December 2015

Revised 21 June 2016

Accepted 22 June 2016

Available online 1 July 2016

\section{Keywords:}

Constitutive integration

Newton methods

Elasto-plasticity

Finite strains

\begin{abstract}
A B S T R A C T
A new semi-implicit stress integration algorithm for finite strain plasticity (compatible with hyperelasticity) is introduced. Its most distinctive feature is the use of different parameterizations of equilibrium and reference configurations. Rotation terms (nonlinear trigonometric functions) are integrated explicitly and correspond to a change in the reference configuration. In contrast, relative Green-Lagrange strains (which are quadratic in terms of displacements) represent the equilibrium configuration implicitly. In addition, the adequacy of several objective stress rates in the semi-implicit context is studied. We parametrize both reference and equilibrium configurations, in contrast with the so-called objective stress integration algorithms which use coinciding configurations. A single constitutive framework provides quantities needed by common discretization schemes. This is computationally convenient and robust, as all elements only need to provide pre-established quantities irrespectively of the constitutive model. In this work, mixed strain/stress control is used, as well as our smoothing algorithm for the complementarity condition. Exceptional time-step robustness is achieved in elasto-plastic problems: often fewer than one-tenth of the typical number of time increments can be used with a quantifiable effect in accuracy. The proposed algorithm is general: all hyperelastic models and all classical elasto-plastic models can be employed. Plane-stress, Shell and 3D examples are used to illustrate the new algorithm. Both isotropic and anisotropic behavior is presented in elasto-plastic and hyperelastic examples.
\end{abstract}

(c) 2016 Elsevier Ltd. All rights reserved.

\section{Introduction}

In retrospective, the implementation of multiple constitutive laws and multiple element formulations limits the choice in terms of finite strain constitutive integration. Distinct constitutive laws should not require distinct finite element implementations and mixed finite element formulations should be able to use any constitutive law implementation. In structural elements, this decoupling was the leitmotiv of, among others, the degenerate shell formulation (cf. [2]) and multiparameter shell formulations (cf. [21]). This also established the strain-driven algorithms as standard (e.g. [39,41,24]) since isoparametric finite elements are displacement-based, strain is directly available. Some important contributions, described in the books by Belytschko, Liu and Moran [15] and Bathe [13] mention the decoupling. In this sense, a

\footnotetext{
* Corresponding author at: Department of Physics, University of Évora, Colégio Luís António Verney, Rua Romão Ramalho, 59, 7002-554 Évora, Portugal.

E-mail address: pmaa@uevora.pt (P. Areias).
}

component perspective on discretization methods was introduced by Areias et al. [11] and a formalization of a general framework based on $\boldsymbol{F}_{e} \boldsymbol{F}_{p}$ decomposition was introduced by Areias et al. [7] after a first work focusing on smoothing the complementarity condition of elasto-plasticity [10]. However, that approach requires the inversion of fourth-order tensors, in contrast with the present contribution.

In this work, we propose a simplification of the finite-strain constitutive algorithms with different parameterizations of equilibrium and reference configurations.

Some considerations are required to contextualize the present work:

1. In many element formulations, stress or strain conditions require the use of a local frame, such as beam and shell elements. Dimensional reduction (either strain, such as in the plane-strain case, or stress, such as plane-stress and shell cases) requires specific treatment in the finite strain case. An in-depth study concerning the incompressibility constraint was 
performed by Antman and Schuricht [3]. For the discrete shell case with the correct thickness extensibility, Hughes and Liu [27] were the first to recognize the need for a specific treatment. We here show a general methodology to treat known stress or strain components in finite strain problems.

2. Large amplitude motions with elements containing rotational degrees-of-freedom can take computational advantage of a equilibrium formulation based on relative strains, circumventing the use of total rotation degrees-of-freedom. Alternatives are more unmanageable, specially in the constrained case (e.g. [18]). It is known that some commercial codes store quaternion parameters to avoid the singularities in large amplitude rotations.

Our consistent updated-Lagrangian method (cf. [6,12,9]) is extended to avoid the storage of rotation matrices.

3. Due to their complementarity form (see, e.g. [31]), plasticity problems often exhibit convergence difficulties for large strain values and severe sensitivity to step size [7].

We here present two measures to attenuate these difficulties: the use of a smoothed complementarity condition and the removal of iterative rotation matrices from the constitutive laws in finite strains.

4. Anisotropic constitutive laws make use of a constitutive frame which must be related to the aforementioned local frame.

Even in the absence of dimensional reduction, a local frame is often required for the representation of anisotropic behavior. Among other relevant properties, the proposed algorithm remains valid for anisotropic hyperelasticity,

5. It is computationally convenient that both hyperelastic and finite-strain elasto-plastic laws are implemented in a unique algorithm and applicable to any discretization scheme.

This technique is introduced here by specializing the frame-ofreference.

6. Classical assumed-strain elements typically do not directly provide the deformation gradient (e.g. [16]), necessary for many constitutive formulations.

An estimated deformation gradient can be calculated from the polar decomposition if an approximate rotation matrix is available. Since a constitutive frame is adopted, the rotation matrix is obtained from this frame in two distinct configurations.

7. Since the seminal contributions of Weber and Anand [45] and Simo [39], Kirchhoff stress tensors (i.e. $\boldsymbol{\tau}=J \boldsymbol{\sigma}$ ) are frequently employed in the yield functions. Two fundamental textbooks on this approach are de Souza Neto et al. [24] and Simo and Hughes [41]. The use of Kirchhoff stress tensor is a computational convenience, as commonly adopted elasto-plastic and hyperelastic models are often quasi-incompressible. This is not the case of porous plasticity or metal elasticity (cf. [9]).

8. Although theoretically identified, see Shutov and Kreißig [38] by means of a thermodynamically-consistent function $\left(\psi_{\mathrm{kin}}\right.$ in [38]), back-stresses are often introduced in a ad-hoc form. Typically, this requires a frame-invariant integration very similar to the hypoelastic formulations. Some experiments were performed by Areias and Rabczuk (cf. [10]).

9. Semi-implicit formulations, where certain quantities are fixed in the flow vector (but not the flow vector itself), cf. $[32,15]$ can lead to substantial savings in constitutive integration. We further extend the semi-implicit algorithm presented in [7] to achieve very large time steps. Consistent linearization of integrated form of objective rates is intricate and computationally expensive, making it a possible candidate for the explicit integration part of the semi-implicit scheme.

In this work, rigid body motions are exactly represented.

A more inclusive approach to constitutive modeling in finite strains, compatible with a variety of finite element discretizations, is henceforth delineated and tested. In summary, Section 2 dis- cusses the constitutive integration algorithm in detail including a test of the adequacy of objective rates in the semi-implicit context, Section 3 presents shell, 2D and 3D examples with both isotropic and anisotropic materials and finally some conclusions are drawn in Section 4.

\section{Constitutive integration in finite strains}

\subsection{Objective rates}

In the context of hypoelastic-based elasto-plasticity, objective time-derivatives of spatial stress measures (either Cauchy, $\boldsymbol{\sigma}$, or Kirchhoff, $\boldsymbol{\tau}=J \boldsymbol{\sigma}$ with $J=\operatorname{det} \boldsymbol{F}$ where $\boldsymbol{F}$ is the deformation gradient) are adopted. A comprehensive description of this approach is performed in Chapters 7 and 8 of Simo and Hughes [41]. The goal is to employ a rate version of Hooke's law for metal plasticity. Objections to such model are known (e.g. [39]) but have lost some strength with the seminal work of Lehmann, cf. [30] who proved the equivalence between a specific corotational time-derivative of the Hencky strain (with the logarithmic spin) and the strain rate D. That work has been extended to establish the equivalence between hypoelasticity with the logarithmic rate and hyperelasticity, cf. [47]. The long standing problem of integrability in hypoelasticity is now solved with the logarithmic rate [49]. However, if the rate version of Hooke's law is maintained, the strong ellipticity condition limits the maximum elastic stretch to the range $[0.21162,1.39561]$ [20], which is only slightly better than with the Jaumann spin. Of course any elastic law could be adopted, but Hooke's law in rate form is computationally attractive. A review paper discussing the use of logarithmic spin in finite strain elastoplasticity discusses many of these points, cf. [48], see also [4].

Two classical forms of reasoning about frame-invariance and classical stress tensors (i.e. Cauchy and Kirchhoff) are based on (i) transport equation (equivalent to the use of Lie derivative) and (ii) rotation by exponential integration of a pre-established spin. Using classical notation (e.g. [26,41]), and focusing on the Kirchhoff stress tensor, these correspond to either:

- Pull-back the Kirchhoff stress, calculate the time-derivative of the result, and push-forward this derivative (this corresponds to the Lie derivative of the Kirchhoff stress or Truesdell rate).

- Rotationally neutralize the Kirchhoff stress (i.e. rotate-back to a fixed reference configuration) so that rigid-body terms are explicatively absent from the time-derivative. Of course, this is a particular case of the pull-back, replacing the deformation gradient with the rotation.

For the pull-back, we use the Kirchhoff stress $\tau$, the second PiolaKirchhoff stress $\boldsymbol{S}$ and the deformation gradient $\boldsymbol{F}$ :

$$
\begin{aligned}
& \tau=\boldsymbol{F S F}^{T} \Longleftrightarrow \\
& \dot{\boldsymbol{\tau}}=\boldsymbol{L} \boldsymbol{\tau}+\boldsymbol{\tau} \boldsymbol{L}^{T}+\underbrace{\boldsymbol{F} \dot{\boldsymbol{S}} \boldsymbol{F}^{T}}_{\tilde{\tau}}
\end{aligned}
$$

where $\dot{\tau}$ is the time-derivative of the Kirchhoff stress and $\check{\boldsymbol{\tau}}=\boldsymbol{F} \dot{\boldsymbol{S}} \boldsymbol{F}^{T}$ is identified as the constitutive or objective time-derivative (cf. [43]). In (1), $\boldsymbol{L}=\dot{\boldsymbol{F}} \boldsymbol{F}^{-1}$ is the velocity gradient, with its symmetric part being identified as strain rate, $\boldsymbol{D}=1 / 2\left(\boldsymbol{L}+\boldsymbol{L}^{T}\right)$ and its skew-symmetric part being given by the vorticity tensor $\boldsymbol{W}=1 / 2\left(\boldsymbol{L}-\boldsymbol{L}^{T}\right)$. For the rotationally neutralized case, rotations (here identified by the tensor $\boldsymbol{R})$ replace the deformation gradient in (1):

$$
\begin{aligned}
& \boldsymbol{\tau} \cong \boldsymbol{R S} \boldsymbol{R}^{T} \Longleftrightarrow \\
& \dot{\tau} \cong \boldsymbol{\Omega} \tau+\tau \boldsymbol{\Omega}^{T}+\underbrace{\boldsymbol{R} \dot{\boldsymbol{S}} \boldsymbol{R}^{T}}_{\tau}
\end{aligned}
$$


Choice of a specific $\boldsymbol{R}$ with $\dot{\boldsymbol{R}}^{T}=\boldsymbol{\Omega}$ in (2) is a matter of requirement for a given application. In (2), $\boldsymbol{\Omega}$, a skew-symmetric tensor compatible with the specific $\boldsymbol{R}$, is called a spin. In addition, in (2), $\stackrel{i}{\boldsymbol{\tau}}=\boldsymbol{R} \dot{\boldsymbol{S}} \boldsymbol{R}^{T}$ is a corotational rate. Several discussions are available in the literature, for example Abbasi and Parsa [1] have compared several spins including Lehmann's [30] logarithmic spin. An important work on spins is the one of Reinhard and Dubey [35] where a rationale for the aforementioned equivalence is described. We note that the use of Kirchhoff stress can be justified in standard metal plasticity applications but not in other cases (for example porosity models of plasticity are compressible). This discussion, despite being standard, motivates the following developments.

\subsection{Parametrization of configurations}

We now consider two configurations ${ }^{1} \Omega_{a}$ and $\Omega_{b}$ identified by time instances $t_{a}$ and $t_{b}$ such that $t_{a} \geqslant t_{b}$. Let the positions of a given point $X$ in configurations $\Omega_{a}$ and $\Omega_{b}$ be $\boldsymbol{x}_{a}=\boldsymbol{x}\left(\xi, t_{a}\right) \in \Omega_{a}$ and $\boldsymbol{x}_{b}=\boldsymbol{x}\left(\xi, t_{b}\right) \in \Omega_{b}$ respectively, with $\xi$ being curvilinear coordinates. Derivatives of $\boldsymbol{x}$ with respect to coordinates $\boldsymbol{\xi}$ are denominated Jacobians $(\boldsymbol{J})$ of the corresponding coordinate transformations:

$\boldsymbol{J}_{a}=\frac{\partial \boldsymbol{x}\left(\xi, t_{a}\right)}{\partial \xi}$

$\boldsymbol{J}_{b}=\frac{\partial \boldsymbol{x}\left(\xi, t_{b}\right)}{\partial \xi}$

Using definitions (3) and (4), the relative deformation gradient is given by:

$\boldsymbol{F}_{a b}=\boldsymbol{J}_{a} \boldsymbol{J}_{b}^{-1}$

Simo (cf. [41] p. 281, Eq. (8.1.13)) derived a one-step scheme for integrating the strain rate in frame 0 (which is not standard). If the explicit version is used ( $\alpha=0$ in [41]), it produces the following relative strain from integration of the strain rate:

$\boldsymbol{e}_{a b}=\frac{1}{2}\left(\boldsymbol{F}_{a b}^{T} \boldsymbol{F}_{a b}-\boldsymbol{I}\right)$

such that

$\boldsymbol{e}_{a b} \cong\left(t_{a}-t_{b}\right) \boldsymbol{D}_{b}$

where $\boldsymbol{D}_{b}$ is the strain rate at $t=t_{b}$. Eq. (6) is specially convenient: it is a quadratic function of the position at configuration $\Omega_{a}$, greatly simplifying the linearization and the extension to the full total Lagrangian formulation. It is however an explicit integration method, with predictable shortcomings in terms of drifting. We can therefore use (6) to determine the classical Green-Lagrange strain $\boldsymbol{e}_{a 0}$ :

$\boldsymbol{e}_{a 0}=\frac{1}{2}\left(\boldsymbol{F}_{a 0}^{T} \boldsymbol{F}_{a 0}-\boldsymbol{I}\right) \Longleftrightarrow$

$\boldsymbol{e}_{a 0}=\boldsymbol{e}_{b 0}+\boldsymbol{F}_{b 0}^{T} \boldsymbol{e}_{a b} \boldsymbol{F}_{b 0}$

We still need to determine the conjugate stresses to the relative strain. We recall that, using a classical result of continuum mechanics, the second Piola-Kirchhoff stress is power-conjugate to the time-derivative of the Green-Lagrange strain. The traditional power argument is employed with the determination of $\boldsymbol{S}_{a b}$ :

$$
\begin{aligned}
\dot{W}_{\text {int }} & =\int_{\Omega_{b}} \operatorname{tr}\left[\boldsymbol{S}_{a b}^{T} \dot{\boldsymbol{e}}_{a b}\right] \mathrm{d} \Omega_{b} \\
& =\int_{\Omega_{0}} \operatorname{tr}\left[\boldsymbol{S}_{a 0}^{T} \dot{\boldsymbol{e}}_{a 0}\right] \mathrm{d} \Omega_{0}
\end{aligned}
$$

\footnotetext{
${ }^{1}$ We use standard notation in continuum mechanics [43].
}

where the trace operator $(\operatorname{tr}[\bullet]=\bullet: I)$ as well as the second PiolaKirchhoff stress $\boldsymbol{S}_{a 0} \equiv \boldsymbol{S}$ were used. It is simple, but intricate, to prove the following relation between the second Piola-Kirchhoff stress $\boldsymbol{S}_{a 0}$ and the relative stress $\boldsymbol{S}_{a b}$ :

$\boldsymbol{S}_{a b}=\frac{1}{J_{b 0}} \boldsymbol{F}_{b 0} \boldsymbol{S}_{a 0} \boldsymbol{F}_{b 0}^{T}$

where $J_{b 0}$ is the determinant of $\boldsymbol{F}_{b 0}$ corresponding to the Jacobian of transformation $0 \mapsto b$ :

$J_{b 0}=\operatorname{det} \boldsymbol{F}_{b 0}=\frac{\mathrm{d} \Omega_{b}}{\mathrm{~d} \Omega_{0}}$

To avoid misinterpretation, in a tensor such as $\boldsymbol{S}_{a b}$ we designate the first index $a$ as identifying the equilibrium configuration $\Omega_{a}$ and the second index $b$ as identifying the reference configuration $\Omega_{b}$. Eq. (11) is generalized as follows:

$\boldsymbol{S}_{a c}=\frac{1}{J_{c b}} \boldsymbol{F}_{c b} \boldsymbol{S}_{a b} \boldsymbol{F}_{c b}^{T}$

with $J_{c b}=\operatorname{det} \boldsymbol{F}_{c b}$. With the exception of hyperelastic laws, most constitutive laws use the Cauchy stress. Yield functions should be based on the Cauchy stress, which typically makes use of the notation $\boldsymbol{\sigma}$. It is straightforward to identify the Cauchy stress as $\boldsymbol{S}_{a a}$ :

$$
\begin{array}{r}
\boldsymbol{\sigma}=\boldsymbol{S}_{a a}=\frac{1}{J_{a 0}} \boldsymbol{F}_{a 0} \boldsymbol{S}_{a 0} \boldsymbol{F}_{a 0}^{T} \Longleftrightarrow \\
\boldsymbol{S}_{a a}=\frac{1}{J_{a b}} \boldsymbol{F}_{a b} \boldsymbol{S}_{a b} \boldsymbol{F}_{a b}^{T}
\end{array}
$$

Using a hypoelastic relation, stress updating is performed additively:

$\boldsymbol{S}_{a b}=\boldsymbol{S}_{b b}+\Delta \check{\boldsymbol{S}}_{a}\left(\boldsymbol{e}_{a b}\right)$

where $\Delta \check{\boldsymbol{S}}_{a}$, the constitutive part of the stress, is a function of the relative strain $\boldsymbol{e}_{a b}$. This will be specified later in Section 2.6 in implicit form. Therefore, in [9], to account for compressible metal plasticity, we showed that, using two configurations $\Omega_{a}$ and $\Omega_{b}$, equilibrium equations can be written using the relative deformation gradient $\boldsymbol{F}_{a b}$ and the corresponding stress $\boldsymbol{S}_{a b}$ where indices $a$ and $b$ identify configurations $\Omega_{a}$ and $\Omega_{b}$, respectively:

$\boldsymbol{\nabla}_{b} \cdot\left(\boldsymbol{F}_{a b} \boldsymbol{S}_{a b}\right)^{T}+J_{a b} \boldsymbol{b}=\mathbf{0}$

where $\nabla_{b}$ is the gradient operator with respect to the position $\boldsymbol{x}_{b} \in \Omega_{b}$ (see Fig. 1):

$\nabla_{b} \equiv \frac{\partial}{\partial \boldsymbol{x}_{b}}$

and $\boldsymbol{b}$ is the body load vector compatible with the Cauchy equations of equilibrium. The relative deformation gradient and Jacobian are respectively defined as:

$\boldsymbol{F}_{a b}=\nabla_{b} \cdot \boldsymbol{x}_{a}$

$J_{a b}=\operatorname{det} \boldsymbol{F}_{a b}$

In addition, the notation $\boldsymbol{F}_{a b}^{-1}=\boldsymbol{F}_{b a}$ holds. Since the matrix forms of $\boldsymbol{S}_{a b}$ and $\boldsymbol{e}_{a b}$ are symmetric, Voigt form can be used with advantages. Using the Voigt form (here identified by the upright bold notation), we write (13) as:

$\mathbf{S}_{a c}=\frac{1}{J_{c b}} \mathcal{V}_{S}\left(\boldsymbol{F}_{c b}\right) \mathbf{S}_{a b}$

where $\boldsymbol{S}_{a c}=\operatorname{Voigt}\left[\boldsymbol{S}_{a c}\right], \mathbf{S}_{a b}=\operatorname{Voigt}\left[\boldsymbol{S}_{a b}\right]$, etc. We note that, omitting indices $c$ and $b, \mathcal{V}_{S}\left(\boldsymbol{F}_{c b}\right)$ can be written, if all 6 stress components are relevant, as: 


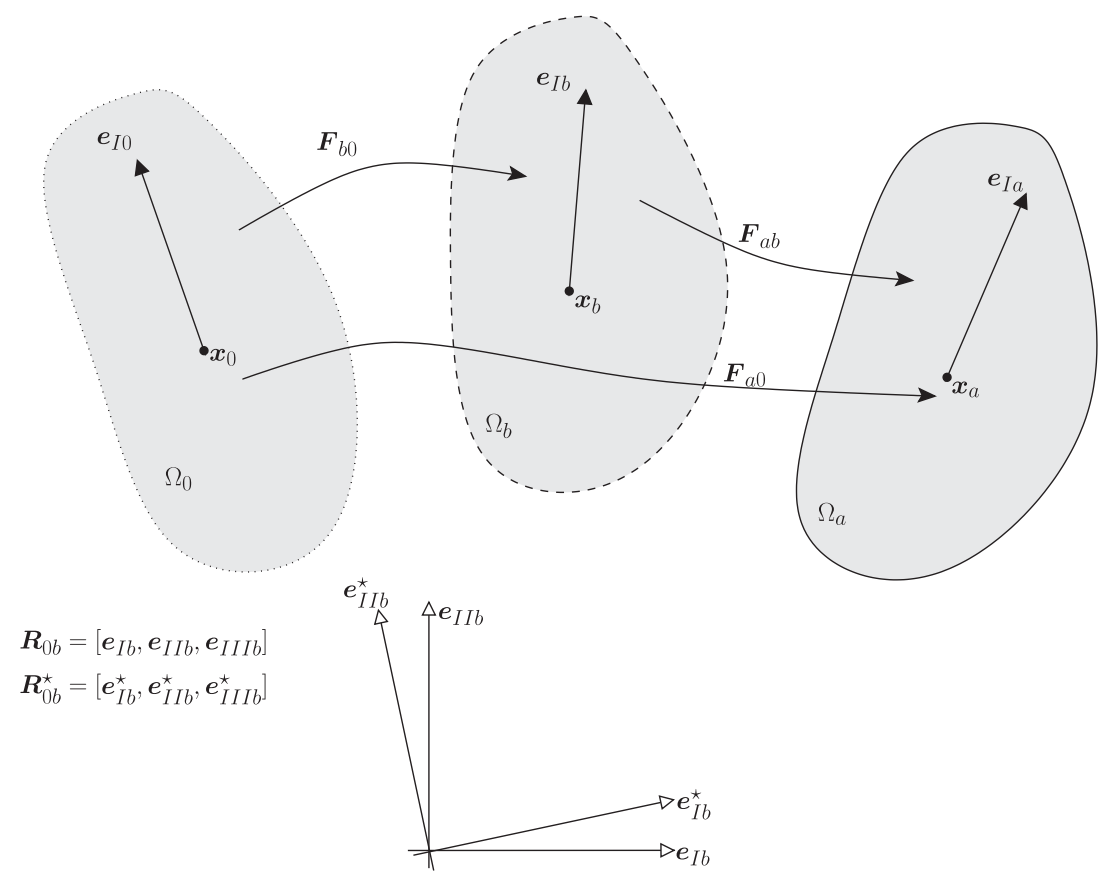

Fig. 1. Moving reference configurations.

$$
\mathcal{V}_{S}(\boldsymbol{F})=\left[\begin{array}{cccccc}
F_{11}^{2} & F_{21}^{2} & F_{31}^{2} & 2 F_{21} F_{11} & 2 F_{31} F_{11} & 2 F_{31} F_{21} \\
F_{12}^{2} & F_{22}^{2} & F_{32}^{2} & 2 F_{22} F_{12} & 2 F_{32} F_{12} & 2 F_{32} F_{22} \\
F_{13}^{2} & F_{23}^{2} & F_{33}^{2} & 2 F_{23} F_{13} & 2 F_{33} F_{13} & 2 F_{33} F_{23} \\
F_{11} F_{12} & F_{21} F_{22} & F_{31} F_{32} & F_{21} F_{12}+F_{11} F_{22} & F_{31} F_{12}+F_{11} F_{32} & F_{31} F_{22}+F_{21} F_{32} \\
F_{11} F_{13} & F_{21} F_{23} & F_{31} F_{33} & F_{21} F_{13}+F_{11} F_{23} & F_{31} F_{13}+F_{11} F_{33} & F_{31} F_{23}+F_{21} F_{33} \\
F_{12} F_{13} & F_{22} F_{23} & F_{32} F_{33} & F_{22} F_{13}+F_{12} F_{23} & F_{32} F_{13}+F_{12} F_{33} & F_{32} F_{23}+F_{22} F_{33}
\end{array}\right]
$$

noting that the Voigt form of stresses follows the convention $\mathbf{S}_{a c}=\left\{S_{11}, S_{22}, S_{33}, S_{12}, S_{13}, S_{23}\right\}_{a c}^{T}$.

We note that Eq. (20) is a non-corotational change of reference configuration for the relative stress. If a relative rotation tensor $\boldsymbol{R}_{c b}$ is used instead of $\boldsymbol{F}_{c b}$, we obtain the following update equation:

$\mathbf{S}_{a c}($ corotational $)=\frac{1}{J_{c b}} \mathcal{V}_{S}\left(\boldsymbol{R}_{c b}\right) \mathbf{S}_{a b}$

The total Green-Lagrange strain is obtained similarly:

$\mathbf{e}_{a c}=\mathbf{e}_{b c}+\mathcal{V}_{E}\left(\boldsymbol{F}_{b c}^{T}\right) \mathbf{e}_{a b}$

where $\mathcal{V}_{E}\left(\boldsymbol{F}_{b c}^{T}\right)=\mathcal{V}_{S}^{T}\left(\boldsymbol{F}_{b c}^{T}\right)$. Chain rule can be applied to the deformation gradient, with the following result:

$\boldsymbol{F}_{a c}=\boldsymbol{F}_{a b} \boldsymbol{F}_{b c}$

Note that Eqs. (22) and (23) are exact, whereas stress updates such as (21) are approximations. For the particular case $\Omega_{c} \equiv \Omega_{0}$, we have $\boldsymbol{F}_{a 0}=\boldsymbol{F}_{a b} \boldsymbol{F}_{b 0}$. Fig. 1 depicts this decomposition. From $\boldsymbol{F}_{a 0}$, we introduce the left Cauchy-Green tensor $\boldsymbol{b}_{a 0}$ as:

$\boldsymbol{b}_{a 0}=\boldsymbol{F}_{a 0} \boldsymbol{F}_{a 0}^{T}$

Spectral decomposition of the positive-definite tensor $\boldsymbol{b}_{a 0}$ reads ( $N s d$ is the number of space dimensions):

$\boldsymbol{b}_{a 0}=\sum_{i=1}^{N s d} \chi_{i} \underbrace{\boldsymbol{n}_{i} \otimes \boldsymbol{n}_{i}}_{\boldsymbol{N}_{i}}$ where $\chi_{i}$ are the eigenvalues of $\boldsymbol{b}_{a 0}, \boldsymbol{n}_{i}$ are the eigenvectors and $\boldsymbol{N}_{i}$ are the eigenprojections. Although not always required, this decomposition (25) is here useful to define the Green-Naghdi rate, based on the polar spin, as well as the logarithmic spin.

\subsection{Frames for local quantities}

Two salient aspects in geometric modeling are described: structural elements, such as beams and shell elements, naturally possess a local frame corresponding to their spatial orientation. Constitutive quantities take advantage of these local frames. In addition, anisotropic materials are defined in terms of principal anisotropic directions (cf. [34]), which can be defined from the local frame. We therefore introduce a local transformation matrix, $\boldsymbol{T}(\theta, \phi)$ which converts the local frame corresponding to to an anisotropic frame (see Fig. 2):

$\boldsymbol{T}(\theta, \phi)=\left[\begin{array}{ccc}\cos (\phi) \cos (\theta) & -\sin (\theta) & \sin (\phi) \cos (\theta) \\ \cos (\phi) \sin (\theta) & \cos (\theta) & \sin (\phi) \sin (\theta) \\ -\sin (\phi) & 0 & \cos (\phi)\end{array}\right]$

where $\theta$ is the angle between $\boldsymbol{e}_{I I b}$ and $\boldsymbol{e}_{I I b}^{\star}$ and $\phi$ is the angle between $\boldsymbol{e}_{I I I b}$ and $\boldsymbol{e}_{I I I b}^{\star}$ (see Fig. 2). We define the anisotropy frame $\boldsymbol{R}_{0 b}^{\star}$ from $\boldsymbol{R}_{0 b}$ and $\boldsymbol{T}(\theta, \phi)$ as:

$\boldsymbol{R}_{0 b}^{\star}=\boldsymbol{R}_{0 b} \boldsymbol{T}(\theta, \phi)$ 


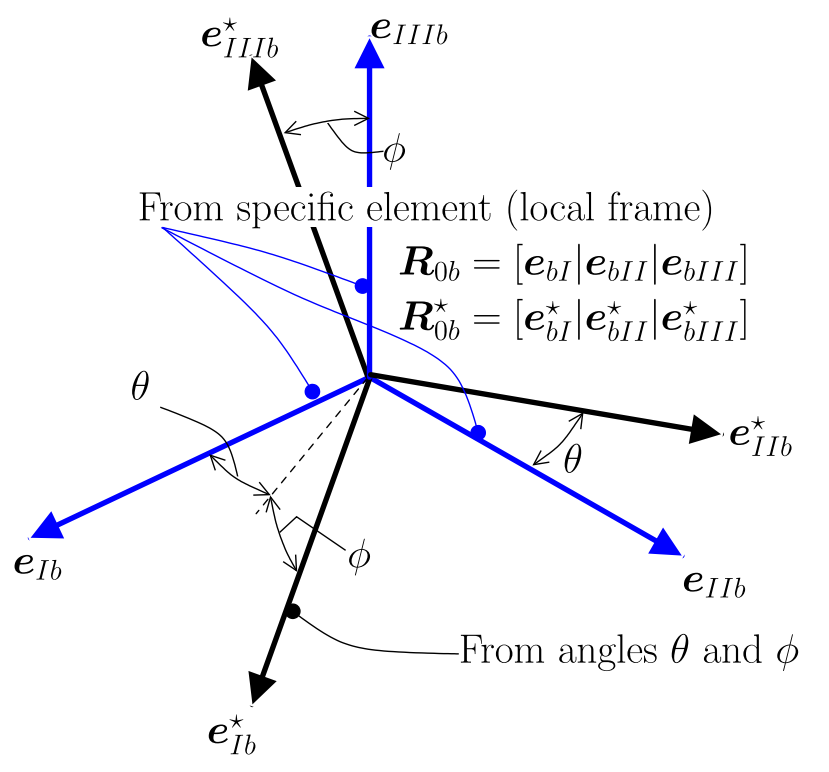

Fig. 2. Relations between frames: $\boldsymbol{R}_{0 b}$ is determined by the specific element and columns of $\boldsymbol{R}_{0 b}^{\star}$ are the principal anisotropic directions. Directions $\boldsymbol{e}_{I}, \boldsymbol{e}_{I I}$ and $\boldsymbol{e}_{I I I}$ are provided by each element technology. Typically in shells $\boldsymbol{e}_{I I I}$ corresponds to the surface normal.

in which the reduced constitutive model is written. Multiplication (27) will result in principal anisotropic directions which are local to each element, which may not be the intended objective. We therefore also introduce the global version, which is:

$\boldsymbol{R}_{0 b}^{\star \star}=\boldsymbol{R}_{0 b} \boldsymbol{R}_{00}^{T} \boldsymbol{T}(\theta, \phi)$

where $\boldsymbol{R}_{00}$ is obtained by evaluating $\boldsymbol{R}_{0 b}$ at $t=0: \boldsymbol{R}_{00}=\left.\boldsymbol{R}_{0 b}\right|_{t_{b}=0}$. Formula (28) defines $\boldsymbol{R}_{0 b}^{\star \star}$ as a global moving frame.

\subsection{Spin integration}

Time integration of spin is now further explored. With that goal, we make use of the velocity gradient. The velocity gradient in equilibrium configuration $\Omega_{a}, \boldsymbol{L}_{a}$, is defined as

$\boldsymbol{L}_{a}=\frac{\partial \dot{\boldsymbol{x}}_{a}}{\partial \boldsymbol{x}_{a}}=\dot{\boldsymbol{F}}_{a b} \boldsymbol{F}_{b a}$

from which the strain rate $\boldsymbol{D}_{a}=\operatorname{Symm}\left[\boldsymbol{L}_{a}\right]$ and the vorticity tensor $\boldsymbol{W}_{a}=\operatorname{Skew}\left[\boldsymbol{L}_{a}\right]$ are determined, respectively, as:

$\boldsymbol{D}_{a}=\frac{1}{2}\left(\boldsymbol{L}_{a}+\boldsymbol{L}_{a}^{T}\right)$

$\boldsymbol{W}_{a}=\frac{1}{2}\left(\boldsymbol{L}_{a}-\boldsymbol{L}_{a}^{T}\right)$

Spins are a function of the vorticity and the decomposition (25). Table 1 shows three common spins, typically used in hypoelastic formulations. It is now established that, for fully implicit algorithms, $\boldsymbol{\Omega}^{\log }$ is the correct spin to ensure compatibility with elasticity [19]. However, for semi-implicit algorithms, no conclusion exists yet. We note that it was recently proved by Shutov and Ihlemann that, in contrast with the Jaummann rate, the Logarithmic rate is not invariant with respect to changes in the reference configuration [37].
Table 1

Common spins.

\begin{tabular}{lll}
\hline Spin name & Symbol & Formula \\
\hline Vorticity (Jaumann) & $\boldsymbol{\Omega}_{a}^{W}$ & $\boldsymbol{W}_{a}$ \\
Polar (Green-Naghdi) & $\boldsymbol{\Omega}_{a}^{R}$ & $\boldsymbol{W}_{a}+\sum_{i=1}^{N s d} \sum_{j \neq i}^{N s d}\left(\frac{\sqrt{\chi_{j}}-\sqrt{\chi_{i}}}{\sqrt{\chi_{i}}+\sqrt{\chi_{j}}}\right) \boldsymbol{N}_{i} \boldsymbol{D}_{a} \boldsymbol{N}_{j}$ \\
Logarithmic & $\boldsymbol{\Omega}_{a}^{\log }$ & $\boldsymbol{W}_{a}+\sum_{i=1}^{N s d} \sum_{j \neq i}^{N s d}\left[\frac{\chi_{i}+\chi_{j}}{\chi_{j}-\chi_{i}}+\frac{2}{\ln \chi_{i}-\ln \chi_{j}}\right] \boldsymbol{N}_{i} \boldsymbol{D}_{a} \boldsymbol{N}_{j}$ \\
With & $\chi_{i} \neq \chi_{j}$ & \\
\hline
\end{tabular}

Time-integration of a spin $\boldsymbol{\Omega}_{a}$ is performed using the two mentioned configurations and provides the following integrated rotation matrix ${ }^{2}$ :

$\boldsymbol{R}_{a b}^{\Omega}=\exp \left[\boldsymbol{\Omega}_{a}\right]$

where

$\exp \left[\boldsymbol{\Omega}_{a}\right]=\boldsymbol{I}+\frac{\sin \left(\Omega_{a}\right)}{\Omega_{a}} \boldsymbol{\Omega}_{a}+\left[\frac{1-\cos \left(\Omega_{a}\right)}{\Omega_{a}^{2}}\right] \boldsymbol{\Omega}_{a}^{2}$

with $\Omega_{a}=\left\|\left\{\left[\Omega_{a}\right]_{32},\left[\Omega_{a}\right]_{13},\left[\Omega_{a}\right]_{21}\right\}\right\|$. To obtain $\boldsymbol{\Omega}_{a}$ using Table 1 , we define $\boldsymbol{W}_{a}$ and $\boldsymbol{D}_{a}$ from $\boldsymbol{F}_{a b}$ as:

$\boldsymbol{W}_{a}=\frac{\operatorname{Skew}\left[\boldsymbol{I}-\boldsymbol{F}_{a b}^{-1}\right]}{t_{a}-t_{b}}$

$\boldsymbol{D}_{a}=\frac{\operatorname{Symm}\left[\boldsymbol{I}-\boldsymbol{F}_{a b}^{-1}\right]}{t_{a}-t_{b}}$

To account for all options of stress integration, we introduce the second-order tensor $\boldsymbol{G}_{a b}$, which is defined as

$\boldsymbol{G}_{a b}= \begin{cases}\boldsymbol{F}_{a b} & \text { Non-corotational } \\ \boldsymbol{R}_{a b}^{W} & \text { Vorticity } \\ \boldsymbol{R}_{a b}^{R} & \text { Polar } \\ \boldsymbol{R}_{a b}^{\text {log }} & \text { Logarithmic }\end{cases}$

\subsection{Integration algorithm}

In terms of algorithm organization, elements with known stress components with conjugate unknown strain components (and vice versa) use an array of size Nvoigt for the Voigt form of strain and stress. When, for a given component, both stress and strain are irrelevant for the discretization method, we omit that component. Beams, plates and shells, besides continuum plane-stress elements have one or more conditions of known stress. Each known stress component corresponds to an unknown strain component and vice versa. Known stresses are identified by the superscript $K$ and for unknown stresses no superscript is required. For simplicity, we also use $K$ for the number of known stress components. Unknown strain components, for example thickness-direction normal strain in a shell, we use the superscript $U$. We provide a tabular representation of these quantities (cf. Table 2) for common discrete models. We note that only the cases Nvoigt $=4$ and $N v$ oigt $=6$ need to be considered for all common discretization cases. A similar introduction to use of full constitutive laws in structural elements was performed by Klinkel and Govindjee [28]. The full algorithm is given in Algorithm 1 with $\Delta \check{\mathbf{S}}_{a b}$ being the constitutive correction in the updated-Lagrangian method.

Certain assumed-strain elements (such as Bathe MITC family

\footnotetext{
${ }^{2}$ Since only relative $\Omega_{a} \rightarrow \Omega_{b}$ quantities are adopted, we do not require a rotation
} update. 
Algorithm 1. Relative Lagrangian formulation (Voigt notation adopted).

Given (calculated) $\boldsymbol{F}_{a b}, \mathbf{e}_{a b}$ (both in frame defined by $\boldsymbol{R}_{0 b}^{\star}$ or $\boldsymbol{R}_{0 b}^{\star \star}$ )

Given (recovered from storage) $\boldsymbol{F}_{b 0}, \mathbf{S}_{b b}^{\star 6}, \mathbf{B}_{b b}, \mathbf{e}_{b 0}$

Transformation and dimension reduction

Relevant Jacobian determinants

$\mathbf{S}_{b b}=\mathcal{R}_{\mathrm{N} v o i g t \times 6} \mathcal{V}_{S}\left(\boldsymbol{R}_{0 A}^{T}\right) \mathbf{S}_{b b}^{\star 6}$

$J_{b 0}=\operatorname{det} \boldsymbol{F}_{b 0}$

Total deformation gradient update

$J_{a b}=\operatorname{det} \boldsymbol{F}_{a b}$

Assignment to equilibrium

$\boldsymbol{F}_{a 0}=\boldsymbol{F}_{a b} \boldsymbol{F}_{b 0}$

stress and backstress

$\mathbf{S}_{a b} \leftarrow \mathbf{S}_{b b}$

Total strain update

$\mathbf{B}_{a b} \leftarrow \mathbf{B}_{b b}$

(UL) Update $\mathbf{S}_{a b} \leftarrow \mathbf{S}_{a b}+\Delta \check{\mathbf{S}}_{a b}, \mathbf{B}_{a b}$ along with sensitivity $\mathcal{C}_{a b}=\frac{\partial \mathbf{S}_{a b}}{\partial \mathbf{e}_{a b}}$

$\mathbf{e}_{a 0}=\mathbf{e}_{b 0}+\mathcal{V}_{E}\left(\boldsymbol{F}_{b 0}^{T}\right) \mathbf{e}_{a b}$

Unknown strain update $\mathbf{e}_{a 0} \leftarrow\left\{\mathbf{e}_{a 0}\right\} \cup\left\{\mathbf{e}_{a 0}^{U}\right\}$

(TL) Determine $\mathbf{S}_{a 0}$ and $\mathbf{B}_{a b}$, along with sensitivity $\mathcal{C}_{a 0}=\frac{\partial \mathbf{S}_{a 0}}{\partial \mathbf{e}_{a 0}}$

Unknown strain update $\mathbf{e}_{a 0} \leftarrow\left\{\mathbf{e}_{a 0}\right\} \cup\left\{\mathbf{e}_{a 0}^{U}\right\}$

(TL) Determine relative stresses

(TL) Determine relative sensitivities

$\mathbf{S}_{a b}=\frac{1}{J_{b 0}} \mathcal{V}_{S}\left(\boldsymbol{F}_{b 0}\right) \mathbf{S}_{a 0}$

Determination of $\boldsymbol{G}_{a b}$

$\mathcal{C}_{a b}=\frac{1}{J_{b 0}} \mathcal{V}_{S}\left(\boldsymbol{F}_{b 0}\right) \mathcal{C}_{a 0} \mathcal{V}_{E}\left(\boldsymbol{F}_{b 0}^{T}\right)$

$\boldsymbol{G}_{a b} \equiv \boldsymbol{G}_{a b}\left(J_{a b}, \boldsymbol{F}_{a b}, \boldsymbol{F}_{a 0}\right)$

Change reference configuration for stresses

(cf. Algorithm 2)

Change reference configuration for back-stresses

$\mathbf{S}_{a a}=\frac{1}{J_{a b}} \mathcal{V}_{S}\left(\boldsymbol{G}_{a b}\right) \mathbf{S}_{a b}$

Determine global stresses

$\mathbf{B}_{a a}=\frac{1}{J_{a b}} \mathcal{V}_{S}\left(\boldsymbol{G}_{a b}\right) \mathbf{B}_{a b}$

$\mathbf{S}_{a a}^{\star 6}=\mathcal{V}_{S}\left(\boldsymbol{R}_{0 A}\right) \mathcal{R}_{N v o i g t \times 6}^{T} \mathbf{S}_{a a}$

Store $\boldsymbol{F}_{a 0}, \mathbf{S}_{a a}^{\star 6}, \mathbf{B}_{a a}, \mathbf{e}_{a 0}$

Return $\mathbf{S}_{a b}$ and $\mathcal{C}_{a b}$

Algorithm 2. Determination of $\boldsymbol{G}_{a b}$.

\begin{tabular}{|c|c|}
\hline \multicolumn{2}{|l|}{ Given $J_{a b}, \boldsymbol{F}_{a b}$ and $\boldsymbol{F}_{a 0}$} \\
\hline Non-corotational & \\
\hline $\begin{array}{l}\text { Relative deformation } \\
\text { gradient }\end{array}$ & $\triangleright \boldsymbol{G}_{a b}=\boldsymbol{F}_{a b}$ \\
\hline \multicolumn{2}{|l|}{ Corotational } \\
\hline $\begin{array}{l}\text { Absolute left Cauchy- } \\
\text { Green tensor }\end{array}$ & $\boldsymbol{b}_{a 0}=\boldsymbol{F}_{a 0} \boldsymbol{F}_{a 0}^{T}$ \\
\hline $\begin{array}{l}\text { Eigenvectors and } \\
\text { eigenvalues of } \boldsymbol{b}_{a 0}\end{array}$ & $\boldsymbol{b}_{a 0}=\sum_{i=1}^{N s d} \chi_{i} \underbrace{\boldsymbol{n}_{i} \otimes \boldsymbol{n}_{i}}_{\boldsymbol{N}_{i}}$ \\
\hline Vorticity & $\boldsymbol{W}_{a}=\frac{1}{2}\left(\boldsymbol{F}_{a b}^{-T}-\boldsymbol{F}_{a b}^{-1}\right)$ \\
\hline Strain rate & $\boldsymbol{D}_{a}=\boldsymbol{I}-\frac{1}{2}\left(\boldsymbol{F}_{a b}^{-T}+\boldsymbol{F}_{a b}^{-1}\right)$ \\
\hline \multirow{3}{*}{$\begin{array}{l}\text { Vorticity rate } \\
\text { Polar }\end{array}$} & $\boldsymbol{\sim} \boldsymbol{G}_{a b}=\exp \left[\boldsymbol{W}_{a}\right]$ \\
\hline & $\boldsymbol{\Omega}_{a}^{R}=\boldsymbol{W}_{a}+\sum_{i=1}^{N s d} \sum_{j \neq i}^{N s d}\left(\frac{\sqrt{x_{j}}-\sqrt{x_{i}}}{\sqrt{\gamma_{i}}+\sqrt{x_{j}}}\right) \boldsymbol{N}_{i} \boldsymbol{D}_{a} \boldsymbol{N}_{j}$ \\
\hline & $\boldsymbol{}_{a b}=\exp \left[\boldsymbol{\Omega}_{a}^{R}\right]$ \\
\hline \multirow[t]{2}{*}{ Logarithmic } & $\boldsymbol{\Omega}_{a}^{\log }=\boldsymbol{W}_{a}+\sum_{i=1}^{N s d} \sum_{j \neq i}^{N s d}\left[\frac{\chi_{i}+\chi_{j}}{\gamma_{j}-\gamma_{i}}+\frac{2}{\ln \gamma_{i}-\ln \gamma_{j}}\right] \boldsymbol{N}_{i} \boldsymbol{D}_{a} \boldsymbol{N}_{j}$ \\
\hline & $\boldsymbol{\sim} \boldsymbol{G}_{a b}=\exp \left[\boldsymbol{\Omega}_{a}^{\log }\right]$ \\
\hline
\end{tabular}

$[25,14])$ do not provide directly a compatible form of $\boldsymbol{F}_{a b}$. A form to approximate $\boldsymbol{F}_{a b}$ in the global frame (here identified as 0 ) consists in using the square root of the right Cauchy-Green tensor, as follows:

$$
\left.\boldsymbol{F}_{a b}\right|_{0} \cong \boldsymbol{R}_{0 a}^{T} \boldsymbol{R}_{0 b} \sqrt{2 \boldsymbol{e}_{a b}+\boldsymbol{I}}
$$

Table 2

Nvoigt and $K$ for common discrete models.

\begin{tabular}{|c|c|c|c|c|c|}
\hline Discrete model & Nvoigt & $K$ & $\mathbf{S}_{a b}, \mathbf{e}_{a b}, \mathbf{S}_{a b}^{\text {reduced }}, \mathbf{e}_{a b}^{\text {reduce }}$ & & $\boldsymbol{R}_{0 b}$ \\
\hline $\begin{array}{l}\text { Shear-deformable } \\
\text { beam }\end{array}$ & 6 & 3 & $\left\{\begin{array}{l}S_{11}^{K}=0 \\
S_{22}^{K}=0 \\
S_{33}^{K} \\
S_{12}^{K}=0 \\
S_{13} \\
S_{23}\end{array}\right\},\left\{\begin{array}{l}e_{11}^{U} \\
e_{22}^{U} \\
e_{33} \\
e_{12}^{U} \\
e_{13} \\
e_{23}\end{array}\right\}$ & $\left\{\begin{array}{l}S_{33} \\
S_{13} \\
S_{23}\end{array}\right\},\left\{\begin{array}{l}e_{33} \\
e_{13} \\
e_{23}\end{array}\right\}$ & $\neg \equiv \boldsymbol{I}$ \\
\hline $\begin{array}{l}\text { Shear-deformable } \\
\text { shell }\end{array}$ & 6 & 1 & $\left\{\begin{array}{l}S_{11} \\
S_{22} \\
S_{33}^{K} \\
S_{12} \\
S_{13} \\
S_{23}\end{array}\right\},\left\{\begin{array}{l}e_{11} \\
e_{22} \\
e_{33}^{U} \\
e_{12} \\
e_{13} \\
e_{23}\end{array}\right\}$ & $\left.\begin{array}{l}S_{11} \\
S_{22} \\
S_{12} \\
S_{13} \\
S_{23}\end{array}\right\},\left\{\begin{array}{l}e_{11} \\
e_{22} \\
e_{12} \\
e_{13} \\
e_{23}\end{array}\right\}$ & $\neg \equiv \boldsymbol{I}$ \\
\hline Plane stress & 4 & 1 & $\left\{\begin{array}{l}S_{11} \\
S_{22} \\
S_{33}^{K}=0 \\
S_{12}^{K}\end{array}\right\},\left\{\begin{array}{l}e_{11} \\
e_{22} \\
e_{33}^{U} \\
e_{12}\end{array}\right\}$, & $\left.\begin{array}{l}S_{11} \\
S_{22} \\
S_{12}\end{array}\right\},\left\{\begin{array}{l}e_{11} \\
e_{22} \\
e_{12}\end{array}\right\}$ & $\equiv \boldsymbol{I}$ \\
\hline Plane strain & 4 & 0 & $\left\{\begin{array}{l}S_{11} \\
S_{22} \\
S_{33} \\
S_{12}\end{array}\right\},\left\{\begin{array}{l}e_{11} \\
e_{22} \\
e_{33}^{K}=0 \\
e_{12}\end{array}\right\}$ & $\left.\begin{array}{l}S_{11} \\
S_{22} \\
S_{12}\end{array}\right\},\left\{\begin{array}{l}e_{11} \\
e_{22} \\
e_{12}\end{array}\right\}$ & $\equiv \boldsymbol{I}$ \\
\hline Three-dimensional & 6 & 0 & $\left\{\begin{array}{l}S_{11} \\
S_{22} \\
S_{33} \\
S_{12} \\
S_{13} \\
S_{23}\end{array}\right\},\left\{\begin{array}{l}e_{11} \\
e_{22} \\
e_{33} \\
e_{12} \\
e_{13} \\
e_{23}\end{array}\right\},\left\{\begin{array}{l}S \\
S \\
S \\
S \\
S \\
S\end{array}\right.$ & $\left.\begin{array}{l}1 \\
2 \\
3 \\
2 \\
3 \\
3\end{array}\right\},\left\{\begin{array}{l}e_{11} \\
e_{22} \\
e_{33} \\
e_{12} \\
e_{13} \\
e_{23}\end{array}\right\}$ & $\equiv \boldsymbol{I}$ \\
\hline Axisymmetric & 4 & 0 & $\left\{\begin{array}{l}e_{11} \\
e_{22} \\
e_{33} \\
e_{12}\end{array}\right\},\left\{\begin{array}{l}S_{1} \\
S_{2} \\
S_{3} \\
S_{1}\end{array}\right.$ & $\left.\begin{array}{l}1 \\
2 \\
3 \\
2\end{array}\right\},\left\{\begin{array}{l}e_{11} \\
e_{22} \\
e_{33} \\
e_{12}\end{array}\right\}$ & $\equiv \boldsymbol{I}$ \\
\hline
\end{tabular}

At this point, we are concerned with the adequacy of classical spins in a semi-implicit algorithm. Simple tests with Algorithms 1 and 2 are performed. An imposed deformation gradient is 

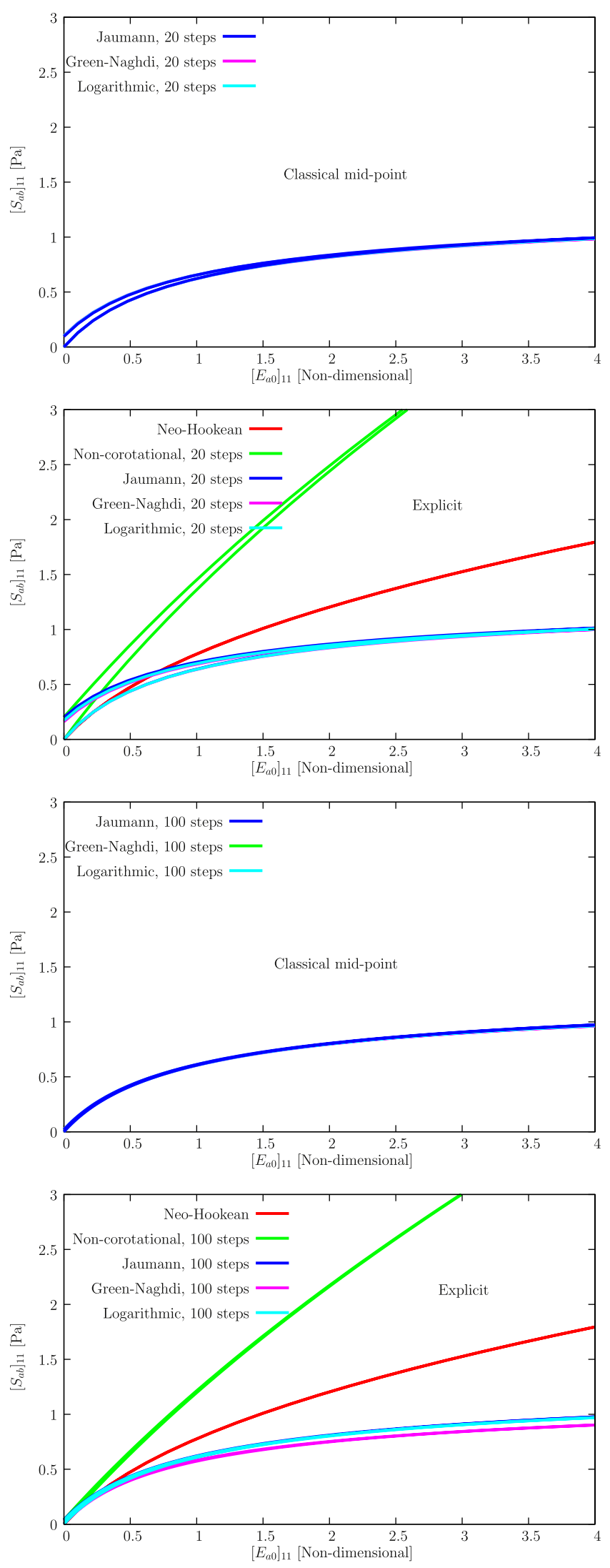

Fig. 3. Effect of choice of $\boldsymbol{G}_{a b}$ in the stress-strain response (case I with unloading).
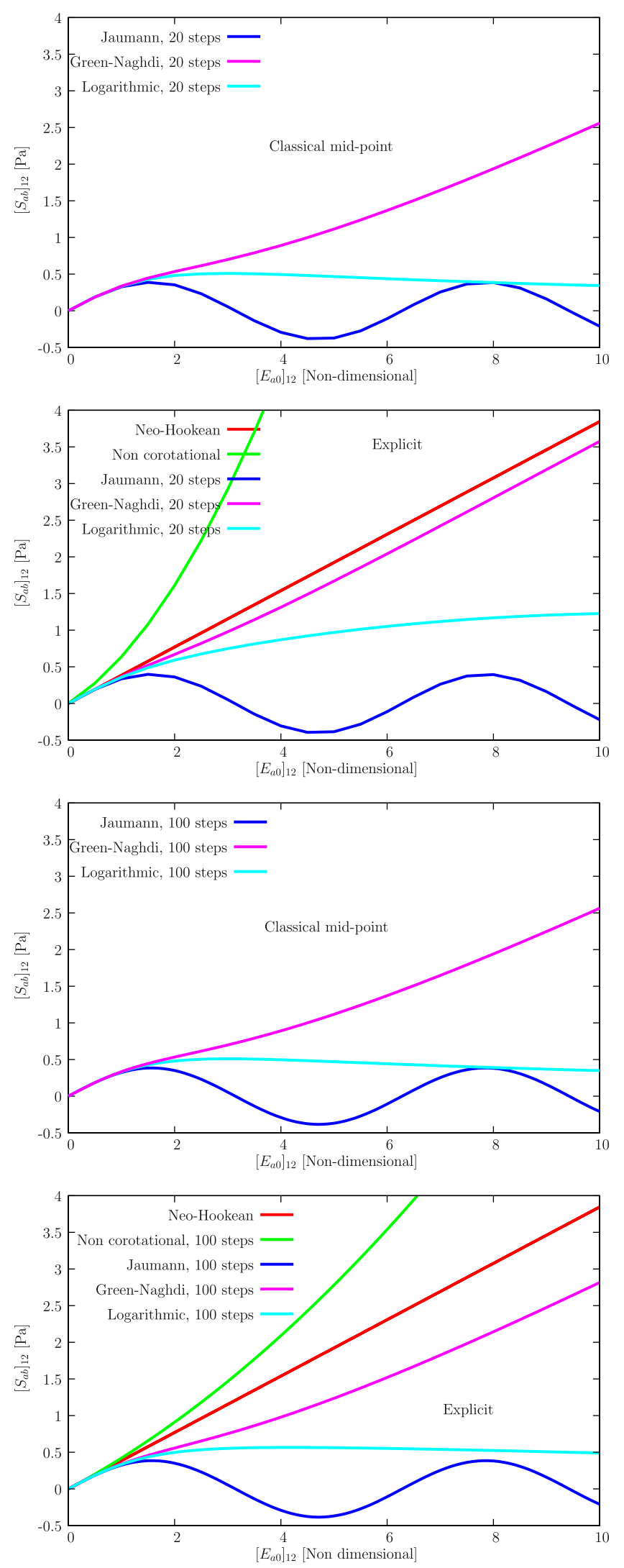

Fig. 4. Effect of choice of $\boldsymbol{G}_{a b}$ in the stress-strain response (case II without unloading). 

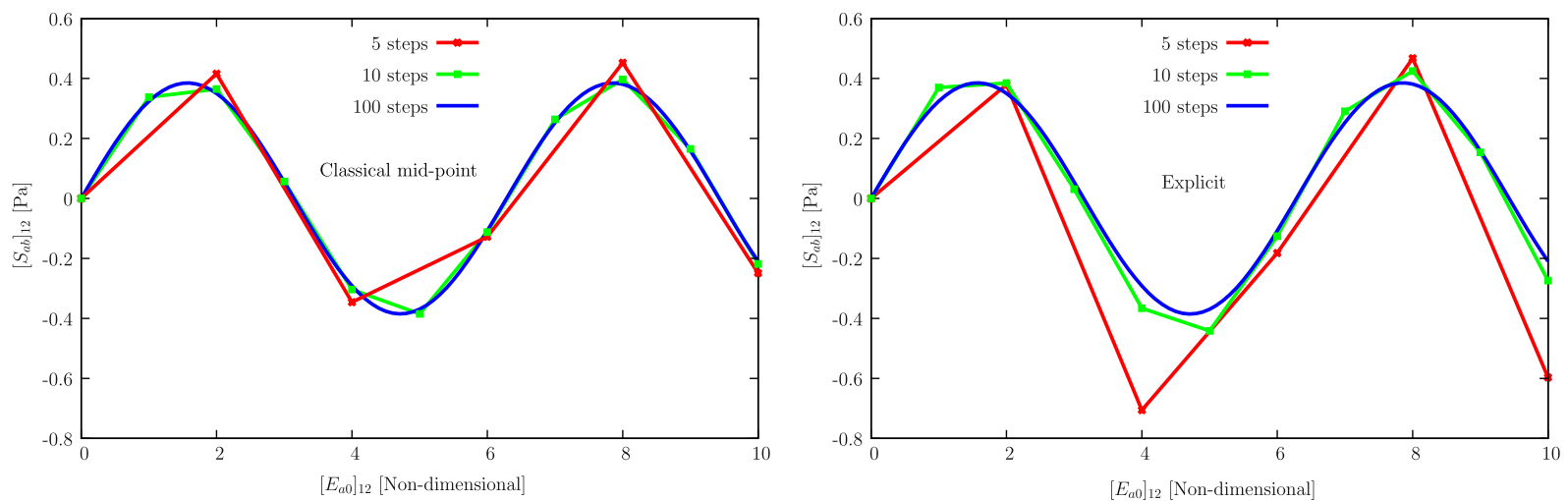

Fig. 5. Effect of step size on the response with the Jaumann rate (case II).

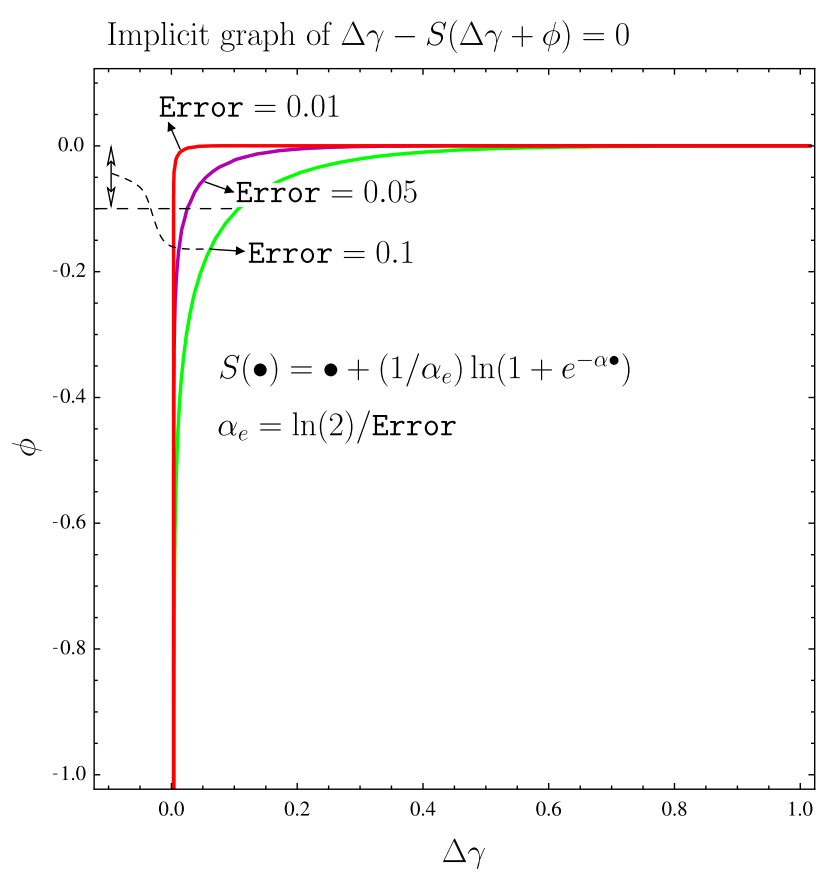

Fig. 6. Replacement of $\mu^{\star} \Delta \gamma-\left\langle\mu^{\star} \Delta \gamma+\phi\right\rangle$ by $\mu^{\star} \Delta \gamma-S\left(\mu^{\star} \Delta \gamma+\phi\right)$ as a function of a Error parameter $\left(\mu^{\star}=1\right.$ is depicted). introduced; for the first test the following imposed deformation gradient is employed:

$\boldsymbol{F}_{I}=\left[\begin{array}{ccc}1+z & -z & 0 \\ 0 & 1 & 0 \\ 0 & 0 & 1\end{array}\right], \quad z \in[0,2] \quad$ and unloading

The second test makes use of the following imposed deformation gradient:

$\boldsymbol{F}_{I I}=\left[\begin{array}{lll}1 & \beta & 0 \\ 0 & 1 & 0 \\ 0 & 0 & 1\end{array}\right], \quad \beta \in[0,5] \quad$ without unloading

For case I, Fig. 3 shows that classical Jaumann, Green-Naghdi (polar) and Logarithmic rate provide similar responses in the fully implicit algorithm with the mid-point rule (cf. [41]) and show very different results in the semi-implicit case. It can be observed that, as the number of steps increases from 20 to 100 , results for all corotational rates become coincident. For case II, Fig. 4 shows that the Green-Naghdi rate, logarithmic rate, the non-corotational rate present significant drifts for small number of steps. Although oscillatory, the Jaumann rate presents only a small difference between 20 and 100 steps and no observable drift. To inspect this in detail, we test the Jaumann rate in both mid-point and semi-implicit cases in Fig. 5. We therefore employ $\boldsymbol{R}_{a b}^{W}$ in the remaining of this work.

Table 3

Prototype yield functions employed.

\begin{tabular}{|c|c|c|}
\hline Yield function & & \\
\hline von-Mises & & $\phi=\frac{\sqrt{\frac{1}{2}\left(S_{11}-S_{22}\right)^{2}+\frac{1}{2}\left(S_{11}-S_{22}\right)^{2}+\frac{1}{2}\left(S_{11}-S_{22}\right)^{2}+3 S_{12}^{2}+3 S_{13}^{2}+3 S_{13}^{2}}}{\sigma_{y}}-1$ \\
\hline Hill & & $\begin{array}{l}\phi=\frac{\sqrt{F\left(S_{22}-S_{33}\right)^{2}+G\left(S_{33}-S_{11}\right)^{2}+H\left(S_{11}-S_{22}\right)^{2}+2 S_{12} S_{12}^{2}+2 S_{13} S_{13}^{2}+2 S_{23} S_{23}^{2}}}{\sigma_{y}}-1 \\
F=\frac{1}{2}\left[\frac{1}{R_{22}^{2}}+\frac{1}{R_{33}^{2}}-\frac{1}{R_{11}^{2}}\right] \\
G=\frac{1}{2}\left[\frac{1}{R_{11}^{2}}+\frac{1}{R_{33}^{2}}-\frac{1}{R_{22}^{2}}\right] \\
H=\frac{1}{2}\left[\frac{1}{R_{11}^{2}}+\frac{1}{R_{22}^{2}}-\frac{1}{R_{33}^{2}}\right] \\
S_{12}=\frac{3}{2 R_{12}^{2}} \\
S_{13}=\frac{3}{2 R_{13}^{2}} \\
S_{23}=\frac{3}{2 R_{23}^{2}}\end{array}$ \\
\hline $\begin{array}{l}\text { Barlat } 91 \\
(\text { BCC: } m=6)\end{array}$ & & $\begin{array}{l}\phi=\frac{\left[\frac{1}{1}\left(T_{1}-T_{2}\right)^{m}+\frac{1}{2}\left(T_{2}-T_{3}\right)^{m}+\frac{1}{2}\left(T_{3}-T_{1}\right)^{m}\right]^{1 / m}}{\sigma_{y}}-1 \\
\text { where } T_{i} \text { are the eigenvalues of } \boldsymbol{T}\end{array}$ \\
\hline $\boldsymbol{T}=\left[\begin{array}{c}\frac{1}{3}\left[P_{3}\left(S_{11}-S_{22}\right)-P_{2}\left(S_{33}-S_{11}\right)\right] \\
P_{6} S_{12} \\
P_{5} S_{13}\end{array}\right.$ & $\begin{array}{cc}P_{6} S_{12} & P_{5} S_{13} \\
\frac{1}{3}\left[P_{1}\left(S_{22}-S_{33}\right)-P_{3}\left(S_{11}-S_{22}\right)\right] & P_{4} S_{23} \\
P_{4} S_{23} & \frac{1}{3}\left[P_{2}\left(S_{33}-S_{11}\right)-P_{1}\left(S_{22}-S_{33}\right)\right]\end{array}$ & \\
\hline
\end{tabular}




\subsection{Mixed strain/stress control in constitutive solution}

Relevant constitutive equations are grouped in a residual vector, here identified by $\boldsymbol{\varphi}$. Constitutive quantities are determined by Newton iteration over this constitutive vector. We therefore reduce the state determination to the root finding with the following nonlinear system:

$\boldsymbol{\varphi}(\underbrace{\overbrace{\mathbf{S}_{a b}}^{\text {Governing }}, \mathbf{e}_{\mathbf{a b}}^{\mathbf{U}}}_{\text {Unknown }} ; \underbrace{\mathbf{S}_{a b}^{K}, \mathbf{e}_{a b}^{K}, \mathbf{e}_{a b}}_{\text {Known }} ; \underbrace{\mathbf{B}_{a b}}_{\text {Back-stresses }}, \underbrace{\boldsymbol{\chi}_{a}}_{\text {Internal var. }})=\mathbf{0}$

where $\mathbf{S}_{a b}^{K}$ is a set of known stresses and $\mathbf{e}_{a b}^{U}$ is the set of unknown strains. Newton iteration for (40) provides the solution for the constitutive unknowns $\mathbf{S}_{a b}, \mathbf{e}_{a b}^{U}, \mathbf{B}_{a b}$ and $\boldsymbol{\chi}_{a}$. A smoothed version of (40) is introduced by using the Chen-Mangasarian replacement functions, which depend on a parameter Error such that (cf. [7]):

$\lim _{\text {Error } \rightarrow 0} \boldsymbol{\varphi}_{\text {Error }}=\boldsymbol{\varphi}$

Newton iteration on (40) provides the following scheme:

$\underbrace{\left[\begin{array}{llll}\frac{\partial \boldsymbol{\varphi}_{\text {Brror }}}{\partial \mathbf{S}_{a b}} & \frac{\partial \boldsymbol{\varphi}_{\text {Brror }}}{\partial \mathbf{e}_{\mathbf{a b}}^{\mathrm{U}}} & \frac{\partial \boldsymbol{\varphi}_{\text {Brror }}}{\partial \mathbf{B}_{a b}} & \frac{\partial \boldsymbol{\varphi}_{\text {Error }}}{\partial \boldsymbol{\chi}_{a}}\end{array}\right]}_{\boldsymbol{J}}\left\{\begin{array}{c}\Delta \mathbf{S}_{a b} \\ \Delta \mathbf{e}_{a b}^{U} \\ \Delta \mathbf{B}_{a b} \\ \Delta \boldsymbol{\chi}_{a}\end{array}\right\}=-\boldsymbol{\varphi}_{\text {Error }}$
After achieving solution using the Newton scheme (42), we calculate the sensitivities from the following equation:

$$
\left\{\begin{array}{c}
\mathrm{d} \mathbf{S}_{a b} \\
\mathrm{~d} \mathbf{e}_{a b}^{U} \\
\mathrm{~d} \mathbf{B}_{a b} \\
\mathrm{~d} \boldsymbol{\chi}_{a}
\end{array}\right\}=-\boldsymbol{J}^{-1}\left[\frac{\partial \boldsymbol{\varphi}_{\text {Error }}}{\partial \mathbf{e}_{\mathbf{a b}}^{\mathbf{K}}} \frac{\partial \boldsymbol{\varphi}_{\text {Error }}}{\partial \mathbf{e}_{a b}}\right]\left\{\begin{array}{l}
\mathrm{d} \mathbf{e}_{a b}^{K} \\
\mathrm{~d} \mathbf{e}_{a b}
\end{array}\right\}
$$

We provide two examples: hyperelasticity in plane-stress and elasto-plasticity with shells. For hyperelasticity in plane-stress, the system is independent of Error:

$$
\boldsymbol{\varphi}\left(\boldsymbol{S}_{a b}, e_{33}^{U} ; S_{33}, \mathbf{e}_{a b}\right)=\left\{\begin{array}{l}
S_{11} \\
S_{22} \\
S_{33}=0 \\
S_{12}
\end{array}\right\}-\frac{\partial \psi\left(\mathbf{e}_{a b}, e_{33}^{U}\right)}{\partial\left\{\begin{array}{l}
e_{11} \\
e_{22} \\
e_{33}^{U} \\
e_{12}
\end{array}\right\}}
$$

For elasto-plasticity in shells we provide a general approach using $\Delta \check{\mathbf{S}}_{a b}=\mathbf{S}_{a b}-\mathbf{S}_{b b}$, with details being given in [7]:

$$
\boldsymbol{\varphi}\left(\boldsymbol{S}_{a b}, e_{33}^{U} ; S_{33}, \mathbf{e}_{a b}\right)=\left\{\begin{array}{l}
\mathbf{e}_{a b}-\mathcal{C}_{\text {linear }}^{-1} \Delta \check{\mathbf{S}}_{a b}-\mathbf{n} \Delta \gamma \\
S_{33} \\
\mu^{\star} \Delta \gamma-\left\langle\mu^{\star} \Delta \gamma+\phi\right\rangle \\
\Delta \boldsymbol{\chi}_{a}-\Delta \gamma \omega\left(\boldsymbol{\chi}_{a}\right)
\end{array}\right\}
$$

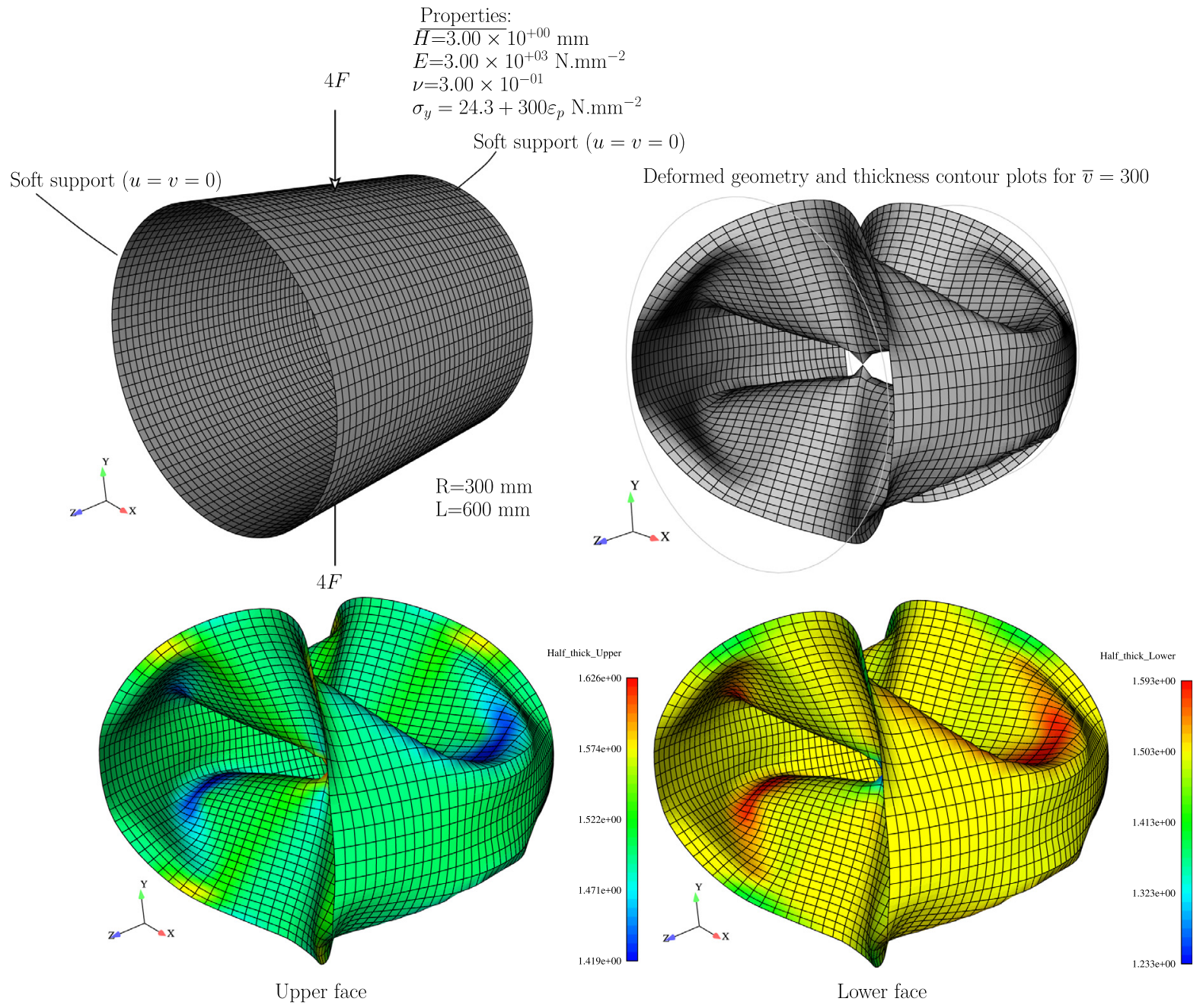

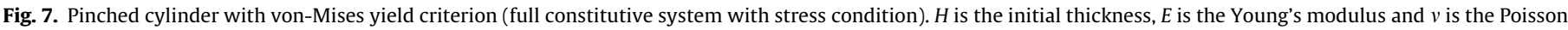
coefficient. The hardening law provides the yield stress $\sigma_{y}$. 
where the Macaulay brackets are used with the usual meaning: $\langle\bullet\rangle=\frac{\bullet \mid+\bullet}{2}$. In (45), $\Delta \gamma$ is the plastic multiplier increment, $\mu^{\star}$ is a dimensional parameter described in [7]. Function $\phi$ in (45) is the yield function and $\omega$ is the vector of internal variable evolution functions. In addition, the flow vector $\mathbf{n}$ is also required for the flow rule, and it is defined as:

$\mathbf{n}=\frac{\partial \phi}{\partial \mathbf{S}_{a b}}$

In (45), equation $\mu^{\star} \Delta \gamma-\left\langle\mu^{\star} \Delta \gamma+\phi\right\rangle$ is a compact form of KuhnTucker (compatibility) conditions. Since (45) is a non-smooth equation system due to the presence of the absolute value function, we use the smooth ramp function $S(\bullet)$ of Chen and Mangasarian [22] for the third equation, which depends on Error:

$\boldsymbol{\varphi}_{\text {Error }}\left(\boldsymbol{S}_{a b}, e_{33}^{U} ; S_{33}, \mathbf{e}_{a b}\right)=\left\{\begin{array}{l}\mathbf{e}_{a b}-\mathcal{C}_{\text {linear }}^{-1} \Delta \check{\mathbf{S}}_{a b}-\mathbf{n} \Delta \gamma \\ S_{33} \\ \mu^{\star} \Delta \gamma-S_{\text {Error }}\left(\mu^{\star} \Delta \gamma+\phi\right) \\ \Delta \boldsymbol{\chi}_{a}-\Delta \gamma \omega\left(\boldsymbol{\chi}_{a}\right)\end{array}\right\}$
Fig. 6 shows the effect of Error in the satisfaction of the complementarity condition. Sensitivities of these constitutive systems are determined by the AceGen [29] add-on to Mathematica [36].

The three prototype yield functions employed are von-Mises, Hill and Barlat 91. All are treated in the same format, without requirement of particular implementations (which are only possible with von-Mises and Hill criteria). The reason for a unique treatment for all yield functions is that any particular yield function is inserted by means of $\phi, \boldsymbol{n}$ and derivatives of $\boldsymbol{n}$. We summarize the yield functions in Table 3.

\section{Numerical examples}

Examples were performed in a in-house code. Element formulations adopted are:

- Mixed quadrilateral shell elements [8].

- B-bar hexahedra [42] with the correct isochoric right CauchyGreen tensor derived by Wriggers [46].

- Traditional displacement-based plane stress triangles.

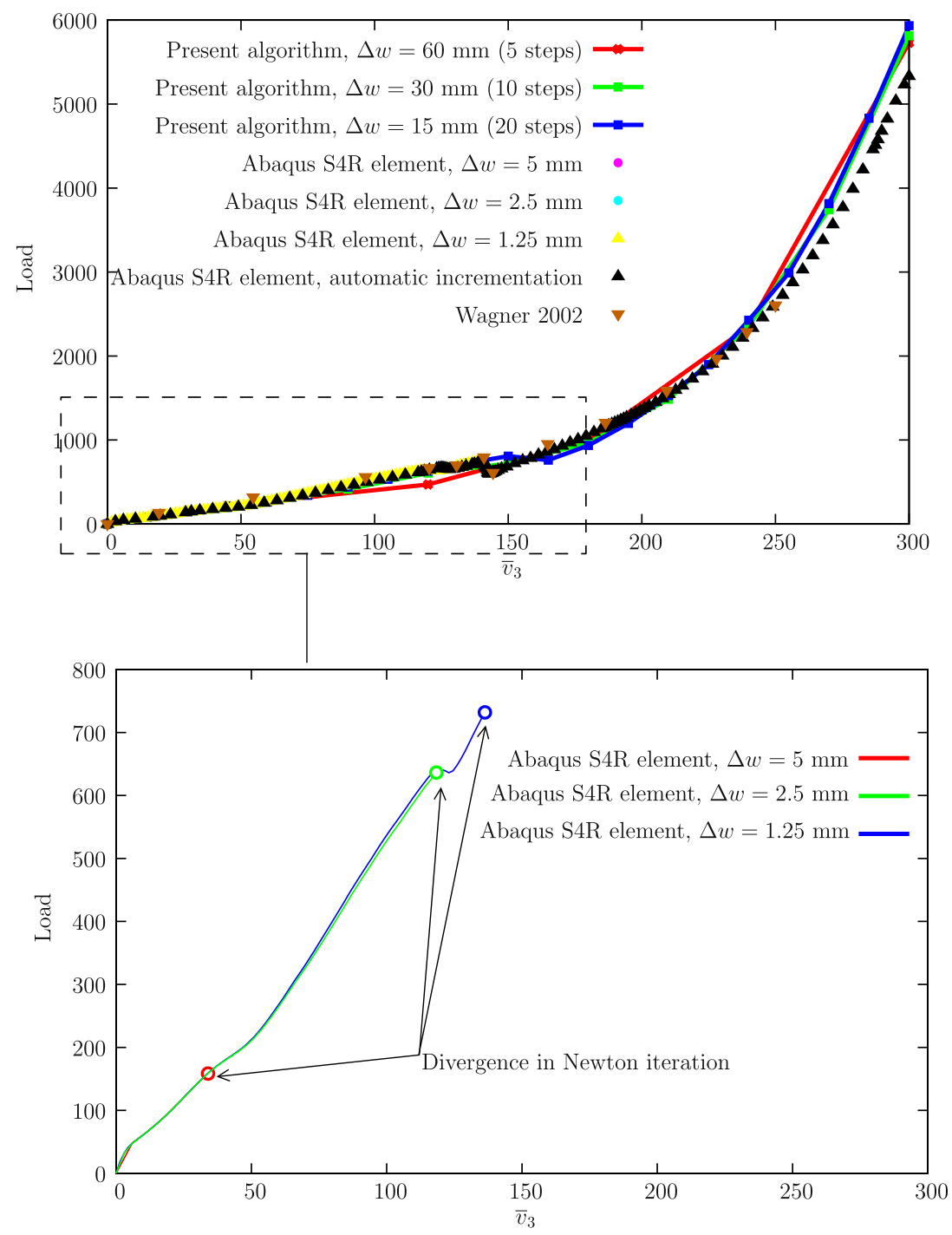

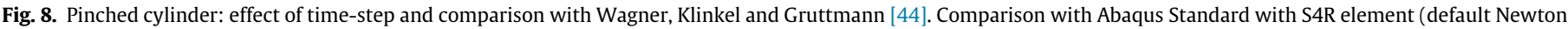
iteration parameters), cf. [23]. 


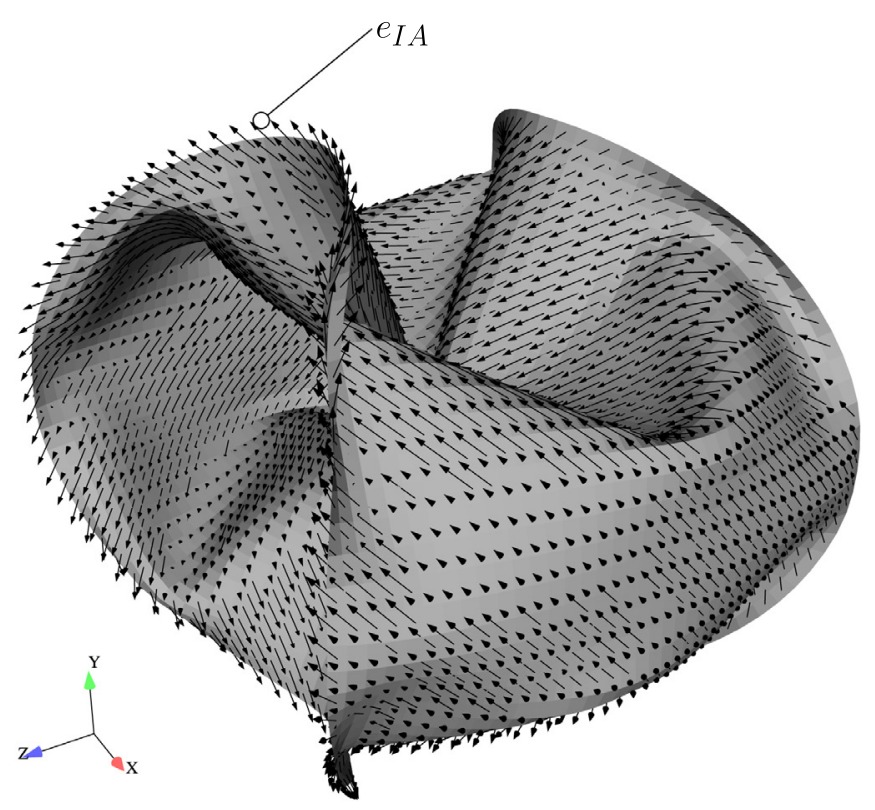

Yield ratio parameters

$$
\begin{aligned}
& R_{11}=1.5 \\
& R_{22}=0.8 \\
& R_{33}=1.0 \\
& R_{12}=1.0 \\
& R_{13}=1.0 \\
& R_{23}=1.0
\end{aligned}
$$

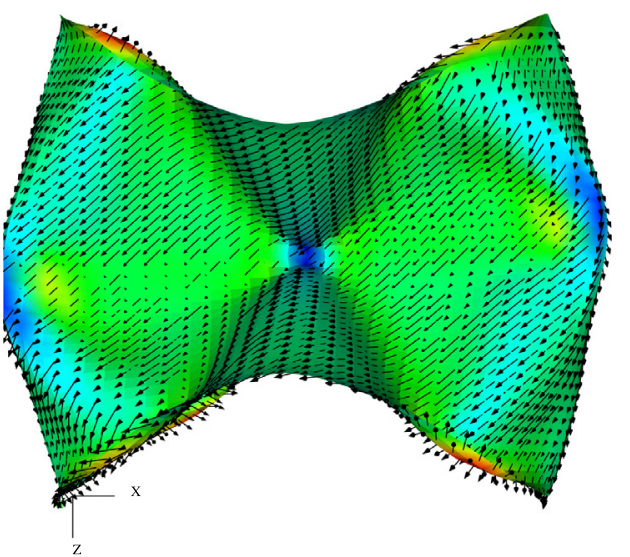

Upper face
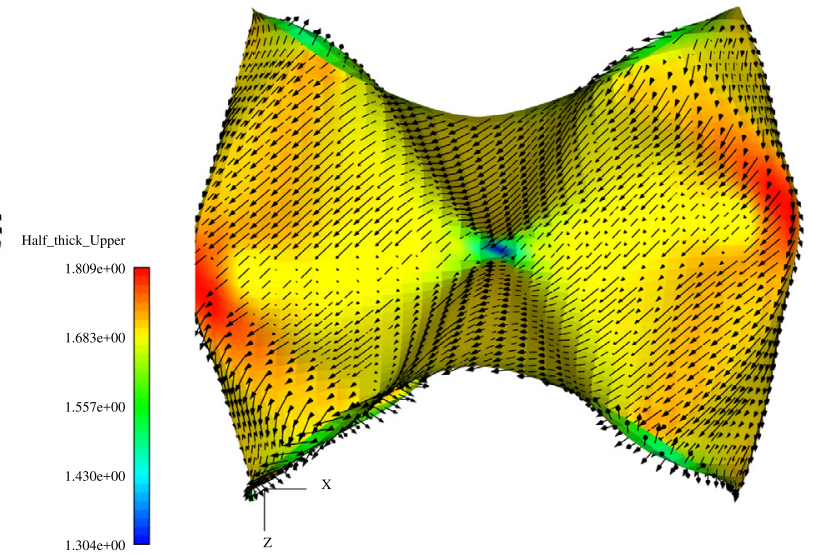

Lower face
Half_thick_Lower

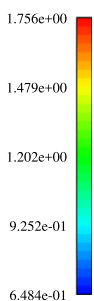

Fig. 9. Pinched cylinder with Hill yield criterion (full constitutive system with stress condition). The anisotropic direction $I$ is shown, as well as a top perspective of the thickness contour plots.

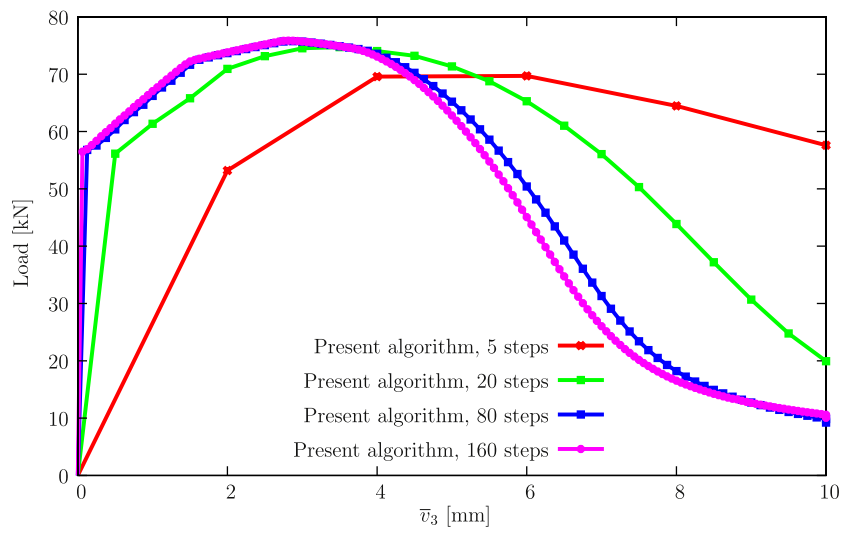

Fig. 10. Pinched cylinder with Hill yield criterion. Step size sensitivity.

In addition, with the exception of the tension test problem, where the effect of Error is assessed, we use Error $=1 \times 10^{-3}$ in all other tests. With the objective of comparing the results with commercially available software, we also use Abaqus Standard [23] version 6.8 with the S4R element for comparison with our algorithm in the isotropic pinched cylinder.

In terms of solution and expected results we have the following:

1. The structural scheme consists of low-order elements (thin shells and 3D) using Gaussian quadrature.

2. Loading scheme is a simple proportional load factor, including deformation-dependent pressure.

3. We measure the drifting, accuracy of results and Newton iteration robustness.

4. Effective plastic strain, $\varepsilon_{p}$ is determined by time-integration $(t)$ up to total time $T$ and power-equivalence (see also [7]):

$\varepsilon_{p}=\int_{0}^{T} \dot{\varepsilon}_{p} \mathrm{~d} t$

where

$\dot{\varepsilon}_{p}=\frac{\mathbf{S}_{a b} \cdot \mathbf{D}_{b}}{\sigma_{y}}$ 


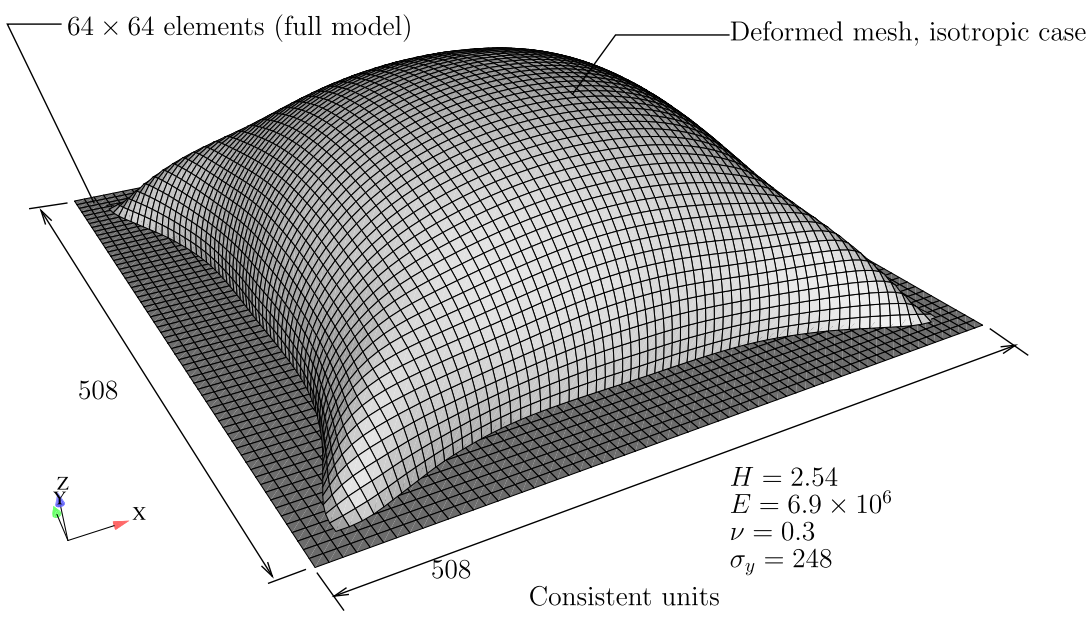

Fig. 11. Square plate under pressure: relevant data.

\subsection{Finite strain plasticity shell problems}

Two classical finite-strain shell problems with thickness extensibility are solved: the pinched cylinder (see Figs. 7 and 9) and the plate under pressure (see Ref. [8] for relevant data as well as Figs. 11 and 12). Besides the classical von-Mises plasticity here implemented with our algorithm (radial return cannot be used, since the plane stress condition invalidates its use), we also test the Hill criterion with local frame angles $\pi / 4$ (pinched cylinder) and $\pi / 6$ (plate under pressure). For the pinched cylinder, excellent results were obtained with a very small number of steps (5), and these agree with Wagner, Klinkel and Gruttmann [44]. A comparison with Abaqus Standard with the S4R element shows that only with variable step size Abaqus achieves convergence for $300 \mathrm{~mm}$ pinching displacement, see Fig. 8. In terms of Newton iteration convergence, robustness of the present algorithm is clear (see also Figs. 9 and 10 for the results with the anisotropic Hill criterion).

For the plate under pressure, Figs. 13 and 14 show some drifting when 5 or 10 steps are used, but overall the results are very robust. Note that it is usual to use 50 or more steps in these problems.

\subsection{Square composite plate}

We now consider a two-layer composite plate, as shown in Fig. 15. For the properties, we use Glass/Epoxy GE-2 material from Table 2.2.1 of Reddy [34]. Fig. 15 shows the relevant data and orthotropic properties for the depicted frame, as well as mesh deformations with the corresponding principal anisotropic directions that follow the deformation. We assess the step-size influence in the results in Fig. 16 to conclude that, for finite strains, a distinct but reasonable (50 or 100) quantity of steps produces very similar results. Note that we purposively employed an incremental orthotropic law to force the use of relative strains. However, this has no visible effect in the results.

\subsection{Tension test of a truncated cone: comparison of von-Mises, Hill and Barlat 91 criteria}

As a prototype model, we perform a test of a nearly-cylindrical specimen originally made of ASTM A-533 steel. It is subject to an imposed displacement in its base. This specific geometry is used to force necking and was adopted by Simo [39] (see also Ref. [40] where the test is explored in further detail) in the context of plas- ticity in principal components, where radial return is still applicable. Test data was obtained by Norris et al. [33] who performed an experimental test and used a 2D finite-difference simulation with grid rezoning in the specimen core. We use the properties of their specimen $2499 R$ and a piece-wise linear law adapted from the one by Simo [39]. Finite element simulations based on a finite-strain version of the radial-return algorithm result in convergence problems after the limit point is reached (cf. [50], pp. 358-359). The geometry, boundary conditions and material properties are summarized in Fig. 17 and Table 4.

Step-size dependence for the von-Mises (Fig. 18), Hill (Fig. 19) and Barlat 91 (Fig. 20) yield functions show that significant drift occurs with a small number of time-steps and gradually disappears when more than 80 steps are employed. Deformed meshes and effective plastic strain for 160 steps are shown in Fig. 21. Of course, if a finer mesh is adopted, some differences occur in the necking region, which are reported in Fig. 22. Constitutive parameter Error is assessed for this problem, and we found that Error $=1 \times 10^{-3}$ is sufficiently accurate for most purposes, see Fig. 23.

\subsection{Plane-stress hyperelastic test with relative Green-Lagrange strains}

A basic rectangular specimen with a circular hole is used to assess the discussed approach in terms of step-size and constraint enforcement (plane stress). The problem data is shown in Fig. 24 along with deformed configurations for the following two classical hyperelastic models employed:

- Kirchhoff/Saint-Venant (cf. [17]):

$$
\begin{aligned}
\boldsymbol{S}_{a 0} & =2 \mu \boldsymbol{e}_{a 0}+\lambda\left(\operatorname{tr} \boldsymbol{e}_{a 0}\right) \boldsymbol{I} \\
\text { with } \mu & =\frac{E}{2(1+v)} \text { and } \lambda=\frac{E v}{(1+v)(1-2 v)}
\end{aligned}
$$

- Transversely isotropic Kirchhoff/Saint-Venant (cf. [17]):

$$
\begin{aligned}
\boldsymbol{S}_{a 0} & =2 \mu \boldsymbol{e}_{a 0}+\lambda\left(\operatorname{tr} \boldsymbol{e}_{a 0}\right) \boldsymbol{I}+2 \beta\left(I_{4}-1\right) \boldsymbol{I} \\
& +2\left[\alpha+\beta\left(I_{1}-3\right)+2 \gamma\left(I_{4}\right)\right] \boldsymbol{a}_{0} \otimes \boldsymbol{a}_{0} \\
& -2 \alpha\left(\boldsymbol{C}_{a 0} \boldsymbol{a}_{0} \otimes \boldsymbol{a}_{0}+\boldsymbol{a}_{0} \otimes \boldsymbol{C}_{a 0} \boldsymbol{a}_{0}\right)
\end{aligned}
$$

with $\boldsymbol{a}_{0}$ being the fiber direction, $I_{1}=\operatorname{tr}\left[\boldsymbol{C}_{a 0}\right], I_{4}=\boldsymbol{a}_{0}^{T} \boldsymbol{C}_{a 0} \boldsymbol{a}_{0}$ and $I_{5}=\boldsymbol{a}_{0}^{T} \boldsymbol{C}_{a 0}^{2} \boldsymbol{a}_{0} . \alpha, \beta$ and $\gamma$ are constitutive properties, cf. Bonet and Burton [17]. These are determined from matrix properties $E_{M}$ and $v_{M}$ and fiber properties $E_{F}$ and $\mu_{F}$. Using consistent units, we have $E=E_{M}=2000, v=v_{M}=0.22, E_{F}=4000$ and $\mu_{F}=500$. Therefore, 

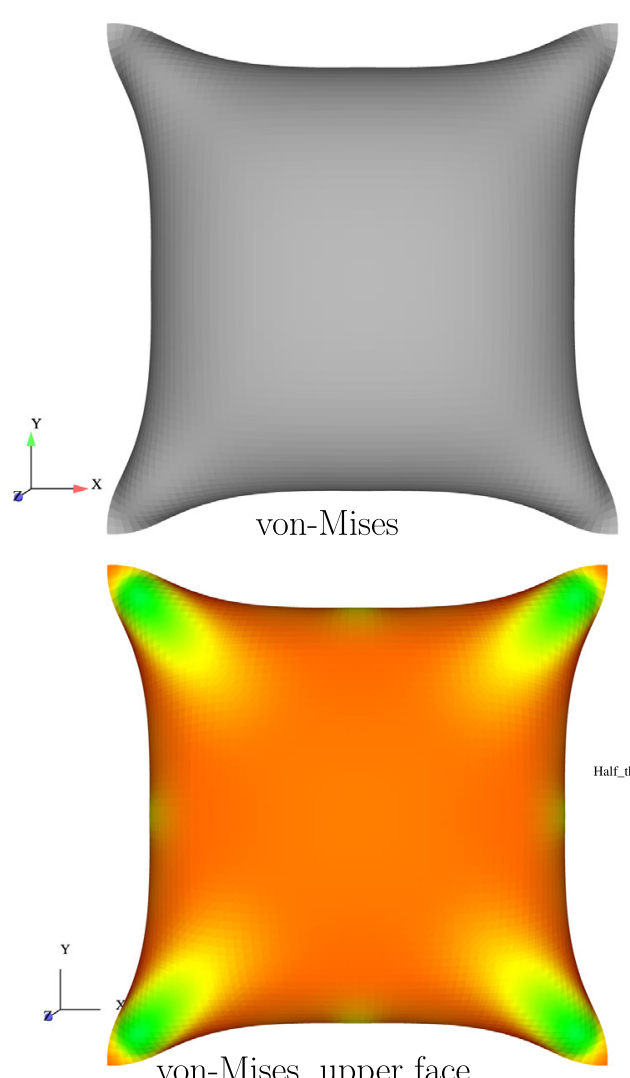

von-Mises, upper face

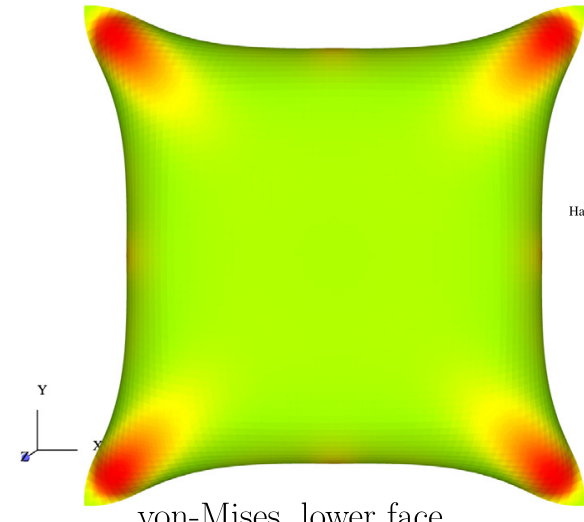

von-Mises, lower face

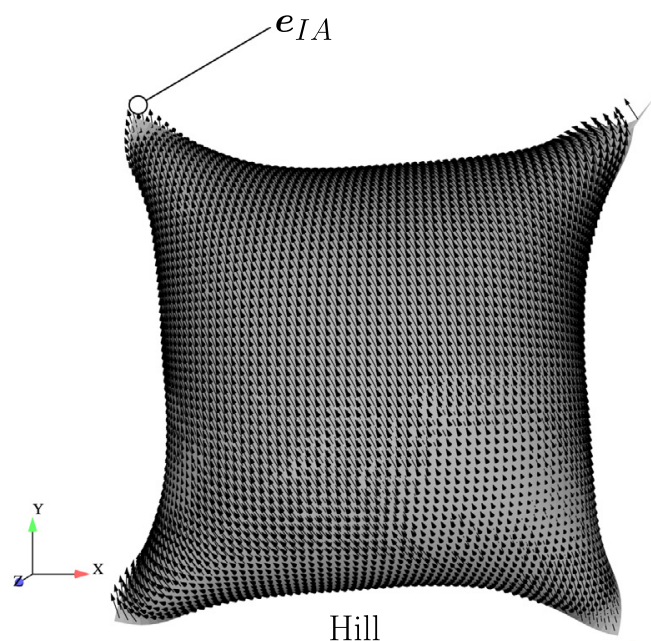

Hill

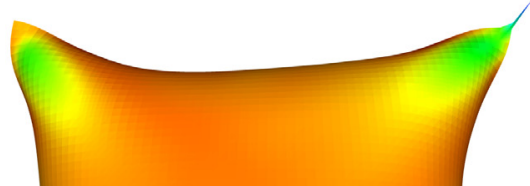

Half_thick_Upper

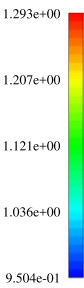

Hill, upper face

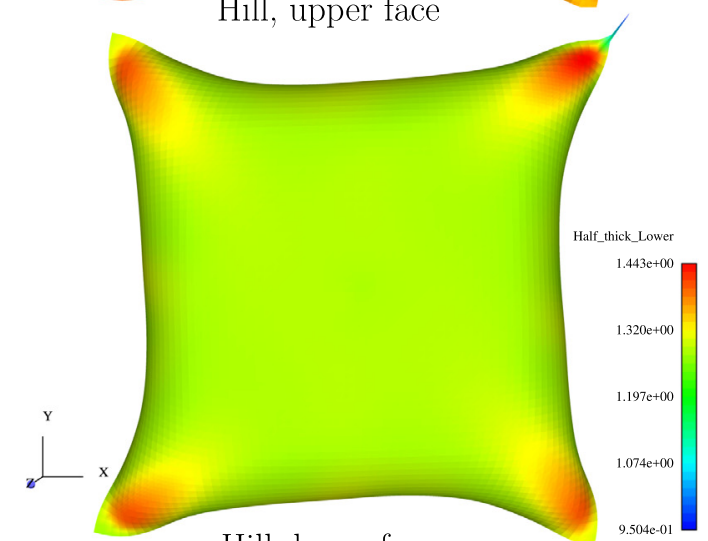

Hill, lower face

Fig. 12. Square plate under pressure: thickness contour plots for von-Mises and Hill criteria.

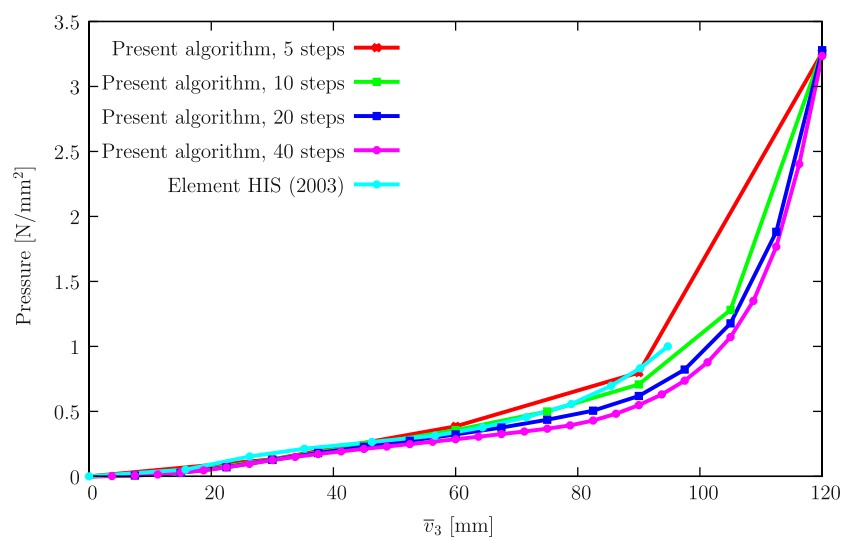

Fig. 13. Square plate under pressure with von-Mises criterion: effect of the time steps. 3D EAS element HIS (cf. [5]) is used for comparison.

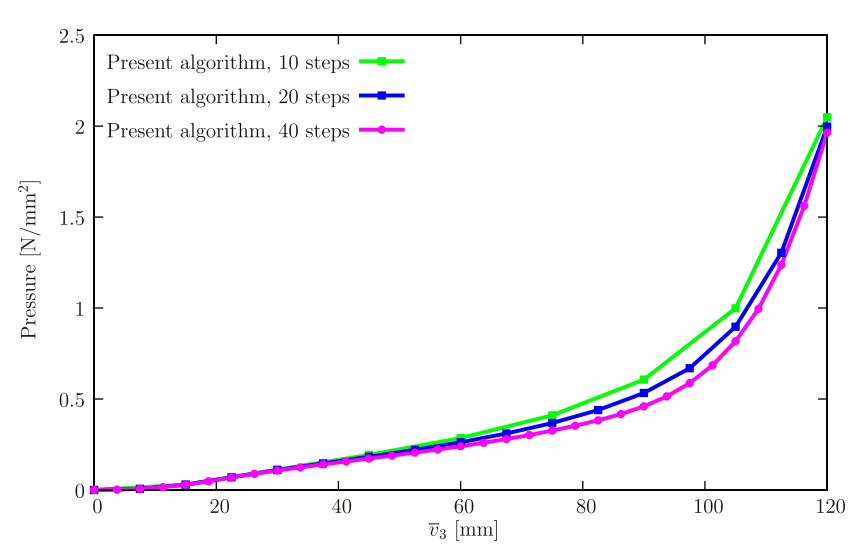

Fig. 14. Square plate under pressure with Hill criterion: effect of the time steps. 


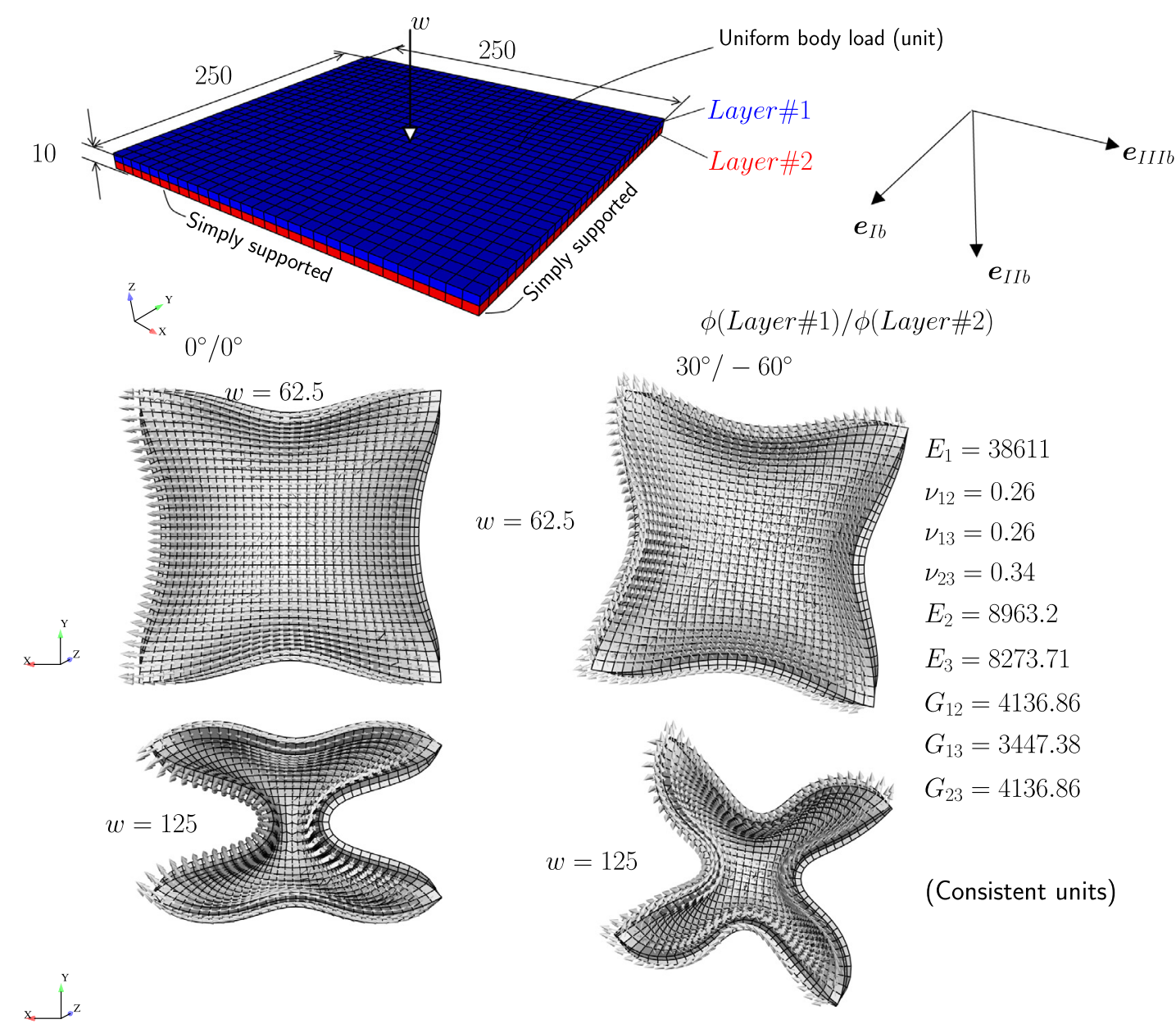

Fig. 15. Two-layer composite plate, relevant data (100 steps were employed for the results shown).

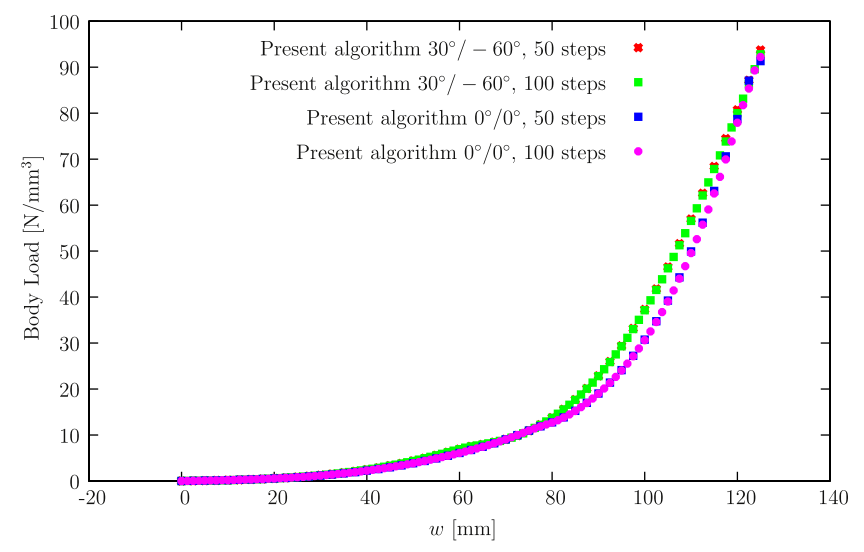

Fig. 16. Two-layer composite plate, effect of step size.

using Eqs. (55a-g) in Bonet and Burton $\alpha=-820, \beta=-33.83$ and $\gamma=4.95$.

Fig. 24 also shows the deformed geometry with the thickness contour plot for a displacement $\bar{u}_{1}=0.08$ in both cases. Although the thickness is updated at the constitutive level, step-size robustness is very high, since the explicit part of the constitutive updating algorithm is not active in the total Lagrangian case, see Fig. 25.

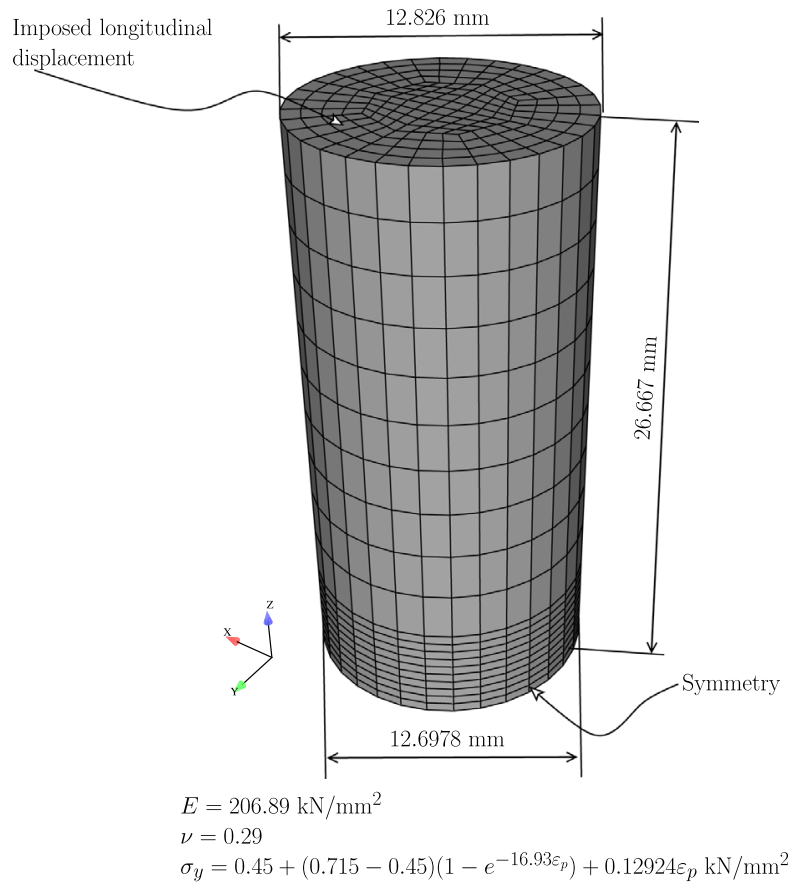

Fig. 17. Relevant dimensions and mesh for the tension test. 
Table 4

Tension test: constitutive properties (consistent units).

\begin{tabular}{ll}
$E$ & 206.9 \\
$v$ & 0.29 \\
$\sigma_{y}$ & $0.45+(0.715-0.45)\left(1-e^{-16.93 \varepsilon_{p}}\right)+0.12929 \varepsilon_{p}$ \\
von-Mises & - \\
Hill & $R_{11}=1.5, R_{22}=0.8, R_{33}=1.0, R_{12}=1.0, R_{13}=1.0, R_{23}=1.0$ \\
Barlat 91 & $a=0.95, b=0.65, c=1.05, f=1.0, g=1.0, h=1.05, m=6$ \\
\hline
\end{tabular}

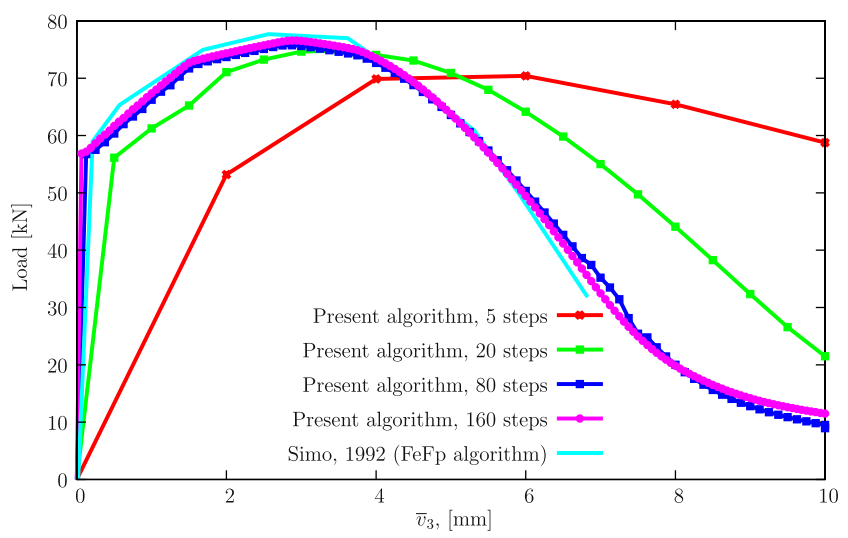

Fig. 18. Results for the tension test: von-Mises criterion.

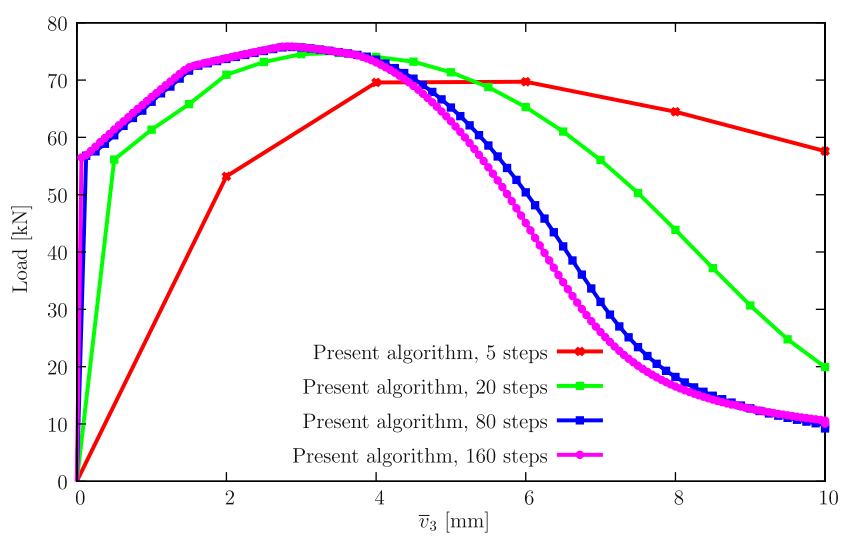

Fig. 19. Results for the tension test: Hill criterion.

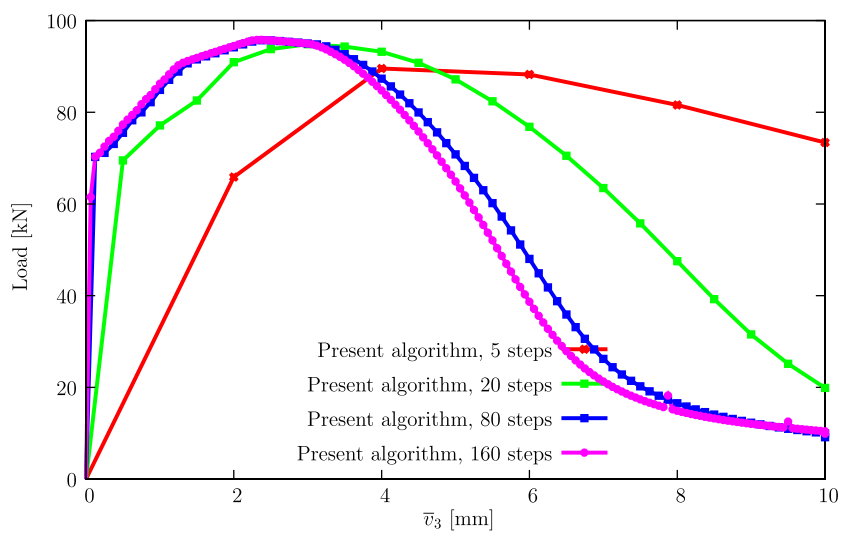

Fig. 20. Results for the tension test: Barlat 91 criterion.
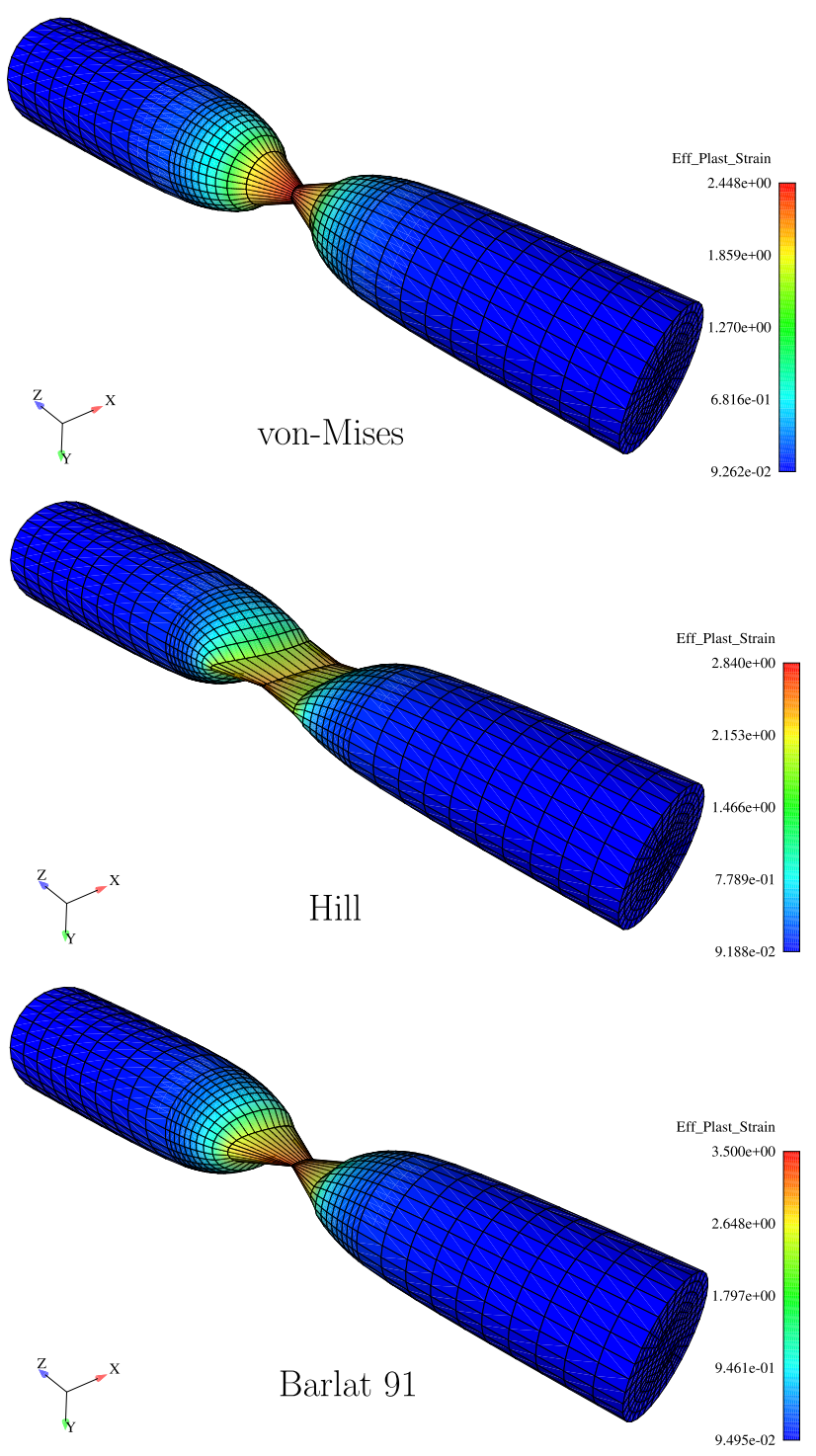

Fig. 21. von-Mises, Hill and Barlat 91 effective plastic strain contour plots.

\section{Conclusions}

An alternative constitutive updating algorithm was proposed, unifying the objective rates and classical total Lagrangian formulations. The algorithm is semi-implicit in the sense that rotations are performed in the end of the time step and therefore do not require linearization. Frame-invariance during time step iterations is ensured by relative Green-Lagrange strains. A comparison of spins was performed to assess the adequacy of the explicit spin integration in terms of time-step sensitivity and it was found that the Jaumann rate provided reasonable results. Very large steps are possible with our algorithm, at the cost of drifting, which is expected from the explicit nature of spin integration. However, the time step sensitivity, for a reasonable number of steps, is similar to the mesh sensitivity in the same problem. In terms of examples, we showed both isotropic and anisotropic problems using, in elasto-plasticity, von-Mises, Hill and Barlat 91 yield functions and, in hyperelasticity, the approaches by Bonet and Burton [17] to use a transversely anisotropic Kirchhoff/Saint-Venant model. An interesting aspect in the total Lagrangian case (which is adopted for 


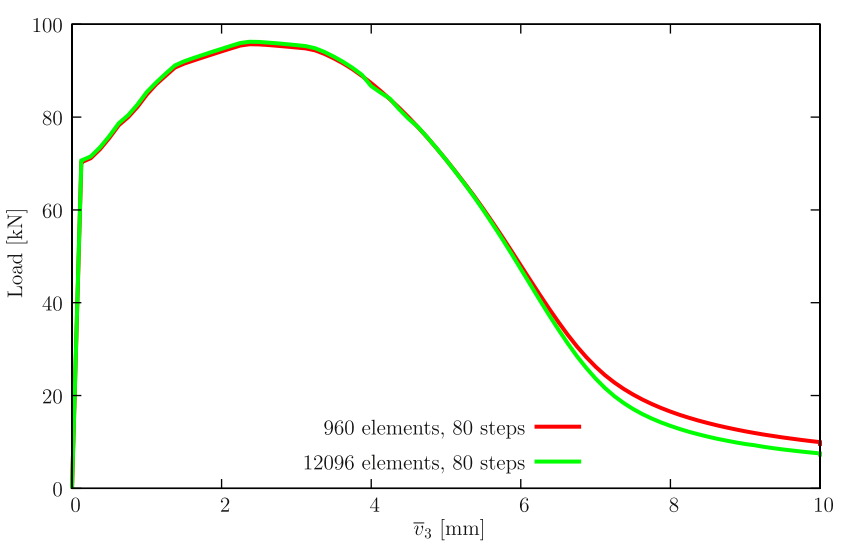

960 elements

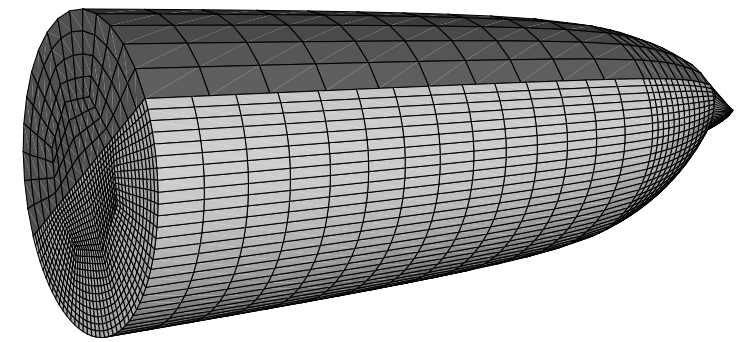

12096 elements<smiles>[V][Te]</smiles>

Fig. 22. Effect of mesh size on the load/displacement results of the tension test specimen (Barlat 91 criterion). The two meshes are superimposed to show the difference in shape.

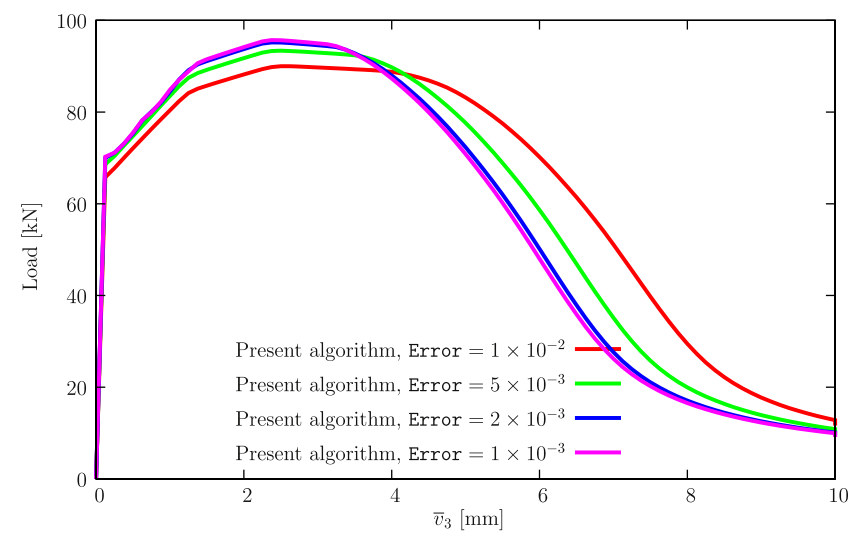

Fig. 23. Effect of Error in the load/displacement results of the tension test specimen (Barlat 91 criterion).

hyperelasticity) is that the relative Green-Lagrange strain is adopted (here denoted by $\boldsymbol{e}_{a b}$ ) and updated to obtain the absolute Green-Lagrange strain $\left(\boldsymbol{e}_{a 0}\right)$. This allows the use of elements formulated always in terms of relative Green-Lagrange strains, which avoids the use of total rotational degrees-of-freedom in shells. Local frames are employed for structural elements, which further allows the determination of the deformation gradient from the strain by a matrix square-root. Therefore, assumed-strain elements such as the MITC family [14] do not require the calculation of the deformation gradient directly. We also tested the logarithmic spin
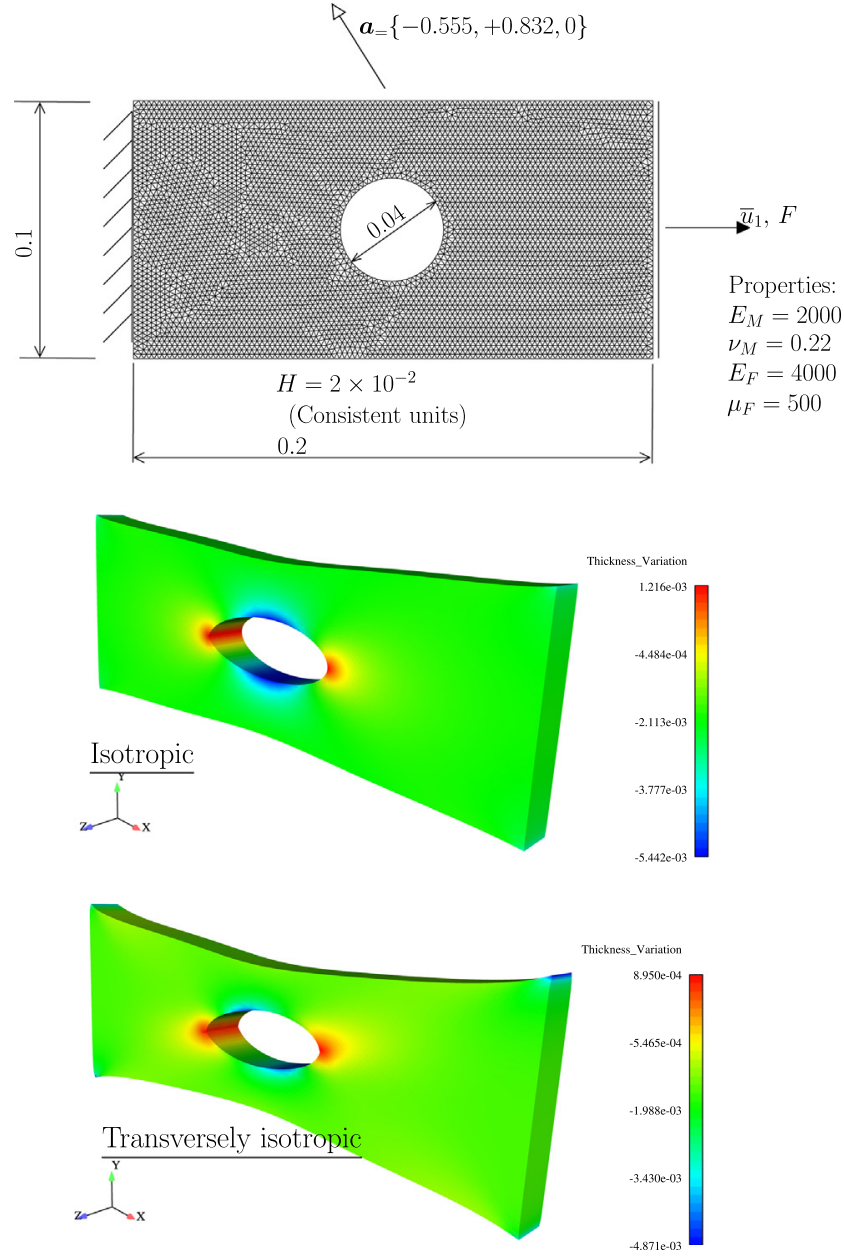

Fig. 24. Plane stress verification test: relevant data and thickness contour plot (5554 nodes and 10742 displacement-based triangles).

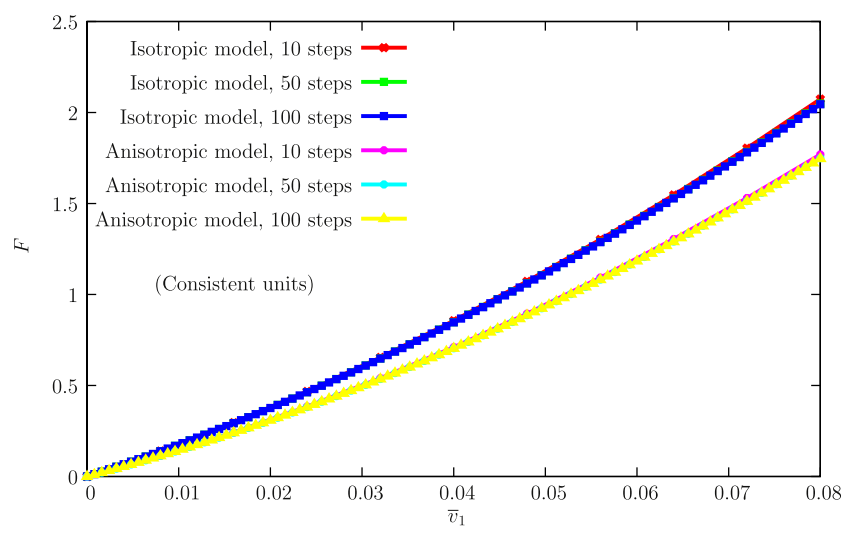

Fig. 25. Step-size sensitivity for isotropic and transversely isotropic Kirchhoff/ Saint-Venant models.

by Lehmann [30] and verified the elasticity preservation in the implicit integration case. However, for the proposed explicit spin integration, significant drifting occurs, which limits the applicability of Lehmann's spin. The clear advantage of our integration algorithm is its wide applicability to both isotropic and anisotropic materials, hyperelastic and elasto-plastic constitutive laws. 


\section{References}

[1] Abbasi B, Parsa MH. Finite element study of the energy dissipation and residual stresses in the closed elastic deformation path. Int J Numer Methods Eng 2006;68:1013-30.

[2] Ahmad S, Irons BM, Zienkiewicz OC. Analysis of thick and thin shell structures by curved finite elements. Int J Numer Methods Eng 1970;2:419-51.

[3] Antman SS, Schuricht F. Incompressibility in rod and shell theories. Math Model Num Anal 1999;33:289-304.

[4] Areias P, Belytschko T. Analysis of finite strain anisotropic elastoplastic fracture in thin plates and shells. J Aerospace Eng 2006;19(4):259-70.

[5] Areias P, César de Sá JMA, Conceição António CA, Fernandes AA. Analysis of 3D problems using a new enhanced strain hexahedral element. Int J Numer Methods Eng 2003;58:1637-82.

[6] Areias P, César de Sá JM, Cardoso R. A simple assumed-strain quadrilateral shell element for finite strains and fracture. Eng Comput 2015;31(4):691-709.

[7] Areias P, Dias-da-Costa D, Pires EB, Infante Barbosa J. A new semi-implicit formulation for multiple-surface flow rules in multiplicative plasticity. Comput Mech 2012;49:545-64.

[8] Areias P, Dias-da Costa D, Pires EB, Van Goethem N. Asymmetric quadrilateral shell elements for finite strains. Comput Mech 2013;52(1):81-97.

[9] Areias P, Dias-da Costa D, Sargado JM, Rabczuk T. Element-wise algorithm for modeling ductile fracture with the Rousselier yield function. Comput Mech 2013:52:1429-43.

[10] Areias P, Rabczuk T. Smooth finite strain plasticity with non-local pressure support. Int J Numer Methods Eng 2010;81:106-34.

[11] Areias P, Rabczuk T, Dias da Costa D, Pires EB. Implicit solutions with consistent additive and multiplicative components. Finite Elem Anal Des 2012;57:15-31.

[12] Areias P, Rabczuk T, Dias-da Costa D. Asymmetric shell elements based on a corrected updated-lagrangian approach. Cmes-Comput Model Eng Sci 2012;88 (6):475-506.

[13] Bathe K-J. Finite element procedures. Prentice-Hall; 1996.

[14] Bathe K-J, Dvorkin EN. A formulation of general shell elements-the use of mixed interpolation of tensorial components. Int J Numer Methods Eng 1986;22:697-722.

[15] Belytschko T, Liu WK, Moran B. Nonlinear finite elements for continua and structures. John Wiley \& Sons; 2000.

[16] Betsch P, Stein E. Numerical implementation of multiplicative elasto-plasticity into assumed strain elements with application to shells at large strains. Comp Method Appl M 1999;179:215-45.

[17] Bonet J, Burton AJ. A simple orthotropic, transversely isotropic hyperelastic constitutive equation for large strain computations. Comp Method Appl M 1998;162:151-64.

[18] Brank B, Ibrahimbegovic A. On the relation between different parametrizations of finite rotations for shells. Eng Comput 2001;18:950-73.

[19] Bruhns OT, Xiao H, Meyers A. Self-consistent Eulerian rate type elastoplasticity models based upon the logarithmic stress rate. Int J Plast 1999:15:479-520.

[20] Bruhns OT, Xiao H, Meyers A. Constitutive inequalities for an isotropic elastic strain-energy function based on Hencky's logarithmic strain tensor. P Roy Soc Lond A Mat 2001:457:2207-26.

[21] Buchter N, Ramm E, Roehl D. Three-dimensional extension of nonlinear shell formulation based on the enhanced assumed strain concept. Int J Numer Methods Eng 1994;37:2551-3568.

[22] Chen C, Mangasarian OL. A class of smoothing functions for nonlinear and mixed complementarity problems. Comput Optim Appl 1996;5:97-138.

[23] Dassault Systèmes, Providence, RI, USA. ABAQUS documentation; 2011.
[24] de Souza Neto EA, Perić D, Owen DRJ. Computational methods for plasticity: theory and applications. West Sussex, England: John Wiley and Sons, Ltd.; 2008.

[25] Dvorkin EN, Bathe KJ. A continuum mechanics based four node shell element for general nonlinear analysis. Eng Comput 1984;1:77-88.

[26] Holzapfel GA. Nonlinear solid mechanics: a continuum approach for engineers. John Wiley and Sons; 2000.

[27] Hughes TJR, Liu WK. Nonlinear finite element analysis of shells: Part I: Threedimensional shells. Comp Method Appl M 1981;26:331-62.

[28] Klinkel S, Govindjee S. Using finite strain 3d-material models in beam and shell elements. Eng Comput 2002;19(8):909-21.

[29] Korelc J. Multi-language and multi-environment generation of nonlinear finite element codes. Eng Comput 2002;18(4):312-27.

[30] Lehmann Th, Guo Z-H, Liang H. The conjugacy between cauchy stress and logarithm of the left stretch tensor. Eur J Mech A-Solid 1991;10(4):395-404.

[31] Lubliner J. Plasticity theory. Macmillan; 1990.

[32] Moran B, Ortiz M, Shih CF. Formulation of implicit finite element methods for multiplicative finite deformation plasticity. Int J Numer Methods Eng 1990;29:483-514.

[33] Norris Jr DM, Moran B, Scudder JK, Quiñones DF. A computer simulation of the tension test. J Mech Phys Solids 1978;26:1-19.

[34] Reddy JN. Mechanics of laminated composite plates and shells. Second ed. Boca Raton: CRC Press; 2004.

[35] Reinhardt WD, Dubey RN. Application of objective rates in mechanical modeling of solids. ASME J Appl Mech 1996;118:692-8.

[36] Wolfram Research Inc. Mathematica; 2007.

[37] Shutov AV, Ihlemann J. Analysis of some basic approaches to finite strain elasto-plasticity in view of reference change. Int J Plast 2014;63:183-97.

[38] Shutov AV, Kreißig R. Application of a coordinate-free tensor formalism to the numerical implementation of a material model. ZAMM 2008;88(11):888-909.

[39] Simo JC. Algorithms for static and dynamic multiplicative plasticity that preserve the classical return mapping schemes of the infinitesimal theory. Comp Method Appl M 1992;99:61-112.

[40] Simo JC, Armero F, Taylor RL. Improved versions of assumed strain tri-linear elements for 3D finite deformation problems. Comp Method Appl M 1993;110:359-86

[41] Simo JC, Hughes TJR. Computational inelasticity. Corrected Second Printing edition. Springer; 2000.

[42] Simo JC, Taylor RL, Pister KS. Variational and projection methods for the volume constraint in finite deformation elasto-plasticity. Comp Method Appl M 1985;51:177-208.

[43] Truesdell C, Noll W. The non-linear field theories of mechanics. third ed. Springer; 2004.

[44] Wagner W, Klinkel S, Gruttmann F. Elastic and plastic analysis of thin-walled structures using improved hexahedral elements. Comput Struct 2002;80:857-69.

[45] Weber G, Anand L. Finite deformation constitutive equations and a time integration procedure for isotropic, hyperelastic-viscoplastic solids. Comp Method Appl M 1990;79:173-202.

[46] Wriggers P. Nonlinear finite element methods. Springer; 2008

[47] Xiao H. Bruhns OT, Meyers A. A natural generalization of hypoelasticity and Eulerian rate type formulation of hyperelasticity. J Elasticity 1999;56:59-93.

[48] Xiao H, Bruhns OT, Meyers A. Elastoplasticity beyond small deformations. Acta Mech 2006;182:31-111.

[49] Xiao H, Bruhns OT, Meyers A. The integrability criterion in finite elastoplasticity and its constitutive implications. Acta Mech 2007;188:227-44.

[50] Zienkiewicz OC, Taylor RL. The finite element method, 5th ed., vol. 2. Butterworth Heinemann; 2000. 